QUARTERLY OF APPLIED MATHEMATICS

VOLUME LXX, NUMBER 2

JUNE 2012, PAGES 311-343

S 0033-569X(2012)01264-X

Article electronically published on February 29, 2012

\title{
ON THE RECONSTRUCTION OF LOW-FREQUENCY MOMENTS IN ACOUSTIC SCATTERING
}

\author{
BY
}

\author{
A. Charalambopoulos
}

Department of Materials Science and Engineering, The University of Ioannina, 45110 Greece

\begin{abstract}
The inverse scattering method via low-frequency moments was introduced several years ago. The specific structure of moments permitted the construction of a linear inversion algorithm that was based on the assumption that the moments were known, at least theoretically. The present work goes deeper and aims at providing a systematic method to reconstruct these moments from measurements. This turns out to be a demanding inverse problem by itself, serving to establish a realistic implementation for the underlying inversion method. It is proved herein how to determine a specific large class of moments. In addition it is proved that not all the moments are able to be determined purely from the set of data. A demanding integral equation methodology is produced to estimate the large class of the remaining moments that are not directly accessible from measurements.
\end{abstract}

1. Introduction. A few years ago, the author contributed to the development and investigation of a novel analytic approach (the linear low-frequency moments method) for the solution of the inverse scattering problem. The proposed technique had been implemented in a series of publications ranging from acoustics [1] to elasticity [2] and from polynomial $[3$ to scatterers with continuous curvature [4]. The basic idea concerned the reconstruction of a polynomial surface or the polynomial approximation of a smooth scatterer via the exploitation of a finite number of generalized moments generated by the leading low-frequency approximation of the total acoustic field and provided by the low-frequency asymptotic analysis of the far-field pattern.

The really striking result consisted of the fact that the exploitation of the Rayleigh approximation alone was sufficient for recovering the shape of the scatterer at least in theoretical terms. It had been proved in that series of papers that taking advantage of all the zero-order low-frequency moments (up to a number firmly dependent on the surface degree) leads to the formation of a linear algebraic system, whose solution provides the coefficients of the representation of the scattering surface in terms of spherical harmonics.

Received September 23, 2010. 2010 Mathematics Subject Classification. Primary 35R30, 76Q05; Secondary 35J05.

(C) 2012 Brown University 
All these years, the linear method of low-frequency moments has been restricted to give exclusively theoretical results. Actually only in [3, the method is subjected to a partial testing in the case of the ellipsoidal scatterer. Even in that case, the data of the inverse scattering problem were not constructed synthetically, neither was the outcome of a measurement process. Instead, the moments were analytically constructed via extended use of integral properties of the ellipsoidal harmonic functions. In addition we pay attention to the work [5], where we find the establishment of a parallel algorithm for solving the inversion scheme along with all the theoretical argumentation concerning the error analysis of the suggested methodology. In general terms, the main idea in all the aforementioned works was to present the manner to reconstruct the scatterer's surface polar representation after the low-frequency moments are given. This approach was of course accompanied with the theoretical establishment of the ability in principle to determine the moments starting from being provided with the low-frequency expansion of the far-field pattern.

The purpose of this work is to form the mechanism connecting the moments with the real data of measurement processes. The first result of this work is the production of a stabilized technique decomposing the low-frequency expansion of the far-field pattern into its coefficients. The second effort is the deconstruction of these far-field coefficients into the low-frequency moments, which theoretically constitute surface integrals of integrands incorporating physical and geometrical characteristics of the scattering problem and can be obtained by simple measurements in special orientations and specific excitations. However, an important by-product is that, apart from theoretical reasoning, not all the moments generated by the Rayleigh approximation alone can be produced immediately and effectively from the knowledge of the far-field pattern. Some of them are strictly connected with moments of higher order, and their separation from this bonding is most demanding and intricate.

More precisely, the moments are divided into two classes: The first one contains surface integrals directly deduced from measurements. The most representative members of this class are the moments disposing of the harmonic kernel and pertaining to the Rayleigh approximation measure alone. However the last members do not exhaust the set of moments directly accessible in measurements. The second class is wider and contains all the moments, which participate in a combined and involved form, whose decomposition is a feasible but demanding process. The investigation of this second class of moments is purely analytic, belongs to the regime of integral equation calculus and introduces the new concept of double moments permitting the estimation of the inaccessible simple moments.

A revised algorithm for the shape reconstruction from low-frequency moments will also be reported in the near future [6]. More precisely, in [6], we will show that in most cases in the framework of the solution of the inverse acoustic scattering problem, it is strongly preferable to abandon the concept of constructing a necessarily linear algebraic system with well-known structural matrix elements, since from the application point of view only a few of these structural moments are deduced directly from data exploitation. There exist very interesting cases of polynomial scatterers with a structural symmetry permitting us to avoid the implication of the so-called inaccessible moments. So in [6], 
we will verify that in many cases the first class of the measurable moments is sufficient for the reconstruction of the shape of the scatterer. However it is always possible to insist in the linearity of the inversion scheme (staying with [3]) but in that case it is proved that moments of both classes are necessary and so the price to pay is the evocation of the heavy integral calculus involving the double moments.

2. Acoustic scattering and the low-frequency moments. We consider the propagation of an acoustic harmonic plane wave inside an infinite three-dimensional acoustic medium hosting an impenetrable inhomogeneity, occupying the bounded region $D$ and being separated by the surrounding space by a soft interface considered to form a starshaped, smooth surface $\partial D$ having at least continuous curvature. The plane wave under discussion is characterized by the time-reduced potential field $u^{i n c}(\mathbf{r} ; \mathbf{k})=\exp (i \mathbf{k} \cdot \mathbf{r})$, which due to the specific time dependence, represents, modulo some multiplicative factor, the pressure field of the incident disturbance as well 7]. Notice that $\mathbf{k}=k \hat{\mathbf{k}}$, where $k$ represents the wave number of the process and the unit vector $\hat{\mathbf{k}}$ indicates the direction of the incidence of the plane wave.

Diffraction of the incident field from the obstacle leads to the creation of the secondary scattering time harmonic wave, with corresponding time-reduced potential field $u^{s c}(\mathbf{r} ; \mathbf{k})$ obeying, in mathematical terms, the following exterior boundary value problem:

$$
\begin{aligned}
\left(\Delta+k^{2}\right) u^{s c}(\mathbf{r} ; \mathbf{k}) & =0, \quad \mathbf{r} \in R^{3} \backslash \bar{D}, \\
u^{s c}(\mathbf{r} ; \mathbf{k})+\exp (i \mathbf{k} \cdot \mathbf{r}) & =0, \quad \mathbf{r} \in \partial D, \\
\frac{\partial}{\partial r} u^{s c}(\mathbf{r} ; \mathbf{k})-i k u^{s c}(\mathbf{r} ; \mathbf{k}) & =O\left(\frac{1}{r^{2}}\right), \quad r=|\mathbf{r}| \rightarrow \infty .
\end{aligned}
$$

The scattered field as well as the incident one satisfies the well-known Helmholtz equation (11) outside the scatterer. The boundary condition (2) reflects the free pressure behavior of the interface, while the radiation Sommerfeld's condition (3) ensures the outgoing orientation of the scattered field and also determines its energy rate at large distances. Furthermore, the scattered field obtains the asymptotic expansion

$$
u^{s c}(\mathbf{r} ; \mathbf{k})=\frac{\exp (i k r)}{i k r} f_{\infty}(\hat{\mathbf{r}} ; \mathbf{k})+O\left(\frac{1}{r^{2}}\right), \quad r=|\mathbf{r}| \rightarrow \infty,
$$

where the normalized scattering amplitude $f_{\infty}(\hat{\mathbf{r}} ; \mathbf{k})$ describes the response of the scatterer in the direction of observation $\hat{\mathbf{r}}$ when it is excited by a plane wave propagating in the direction $\hat{\mathbf{k}}=\mathbf{k} / k$.

The total acoustic field $u^{\text {tot }}=u^{i n c}+u^{s c}$ satisfies the integral representation formula [3]

$$
u^{t o t}(\mathbf{r} ; \mathbf{k})=\exp (i \mathbf{k} \cdot \mathbf{r})-\int_{\partial D} G\left(\mathbf{r}, \mathbf{r}^{\prime}\right) \frac{\partial u^{t o t}}{\partial n^{\prime}}\left(\mathbf{r}^{\prime} ; \mathbf{k}\right) d s\left(\mathbf{r}^{\prime}\right), \quad \mathbf{r} \in R^{3} \backslash D,
$$

where $G\left(\mathbf{r}, \mathbf{r}^{\prime}\right)=\exp \left(i k\left|\mathbf{r}-\mathbf{r}^{\prime}\right|\right) /\left(4 \pi\left|\mathbf{r}-\mathbf{r}^{\prime}\right|\right)$ stands for the free space Green's function of the Helmholtz equation and $\frac{\partial}{\partial n}$ is the normal derivative with respect to the outward normal unit vector on $\partial D$. 
The representation (55) yields the following integral formula for the scattering amplitude:

$$
f_{\infty}(\hat{\mathbf{r}} ; \mathbf{k})=\frac{k}{4 \pi i} \int_{\partial D} \exp \left(-i k \hat{\mathbf{r}} \cdot \mathbf{r}^{\prime}\right) \frac{\partial u^{t o t}}{\partial n^{\prime}}\left(\mathbf{r}^{\prime} ; \mathbf{k}\right) d s\left(\mathbf{r}^{\prime}\right), \quad \hat{\mathbf{r}} \in S^{2},
$$

where $S^{2}$ denotes the unit sphere in $R^{3}$.

The low-frequency treatment $([3],[8,[9],[10])$ of the scattering problem under discussion reduces the above exterior boundary value problem to an infinite sequence of exterior boundary value problems for the Laplace operator, which can be solved iteratively. Specifically, the total field assumes the expansion

$$
u^{t o t}(\mathbf{r} ; \mathbf{k})=\sum_{n=0}^{\infty} \frac{(i k)^{n}}{n !} \Phi_{n}(\mathbf{r} ; \hat{\mathbf{k}})
$$

The $n^{\text {th }}$ order low-frequency approximation $\Phi_{n}(\mathbf{r} ; \hat{\mathbf{k}})$ vanishes on the scatterer's surface $\partial D$ and satisfies the integral representation

$$
\Phi_{n}(\mathbf{r} ; \hat{\mathbf{k}})=(\hat{\mathbf{k}} \cdot \mathbf{r})^{n}-\frac{1}{4 \pi} \sum_{\rho=0}^{n}\left(\begin{array}{l}
n \\
\rho
\end{array}\right) \int_{\partial D}\left|\mathbf{r}-\mathbf{r}^{\prime}\right|^{\rho-1} \frac{\partial \Phi_{n-\rho}}{\partial n^{\prime}}\left(\mathbf{r}^{\prime} ; \hat{\mathbf{k}}\right) d s\left(\mathbf{r}^{\prime}\right), \quad \mathbf{r} \in R^{3} \backslash D .
$$

The scattering amplitude is then expressed via the low-frequency approximations as follows:

$$
\begin{aligned}
f_{\infty}(\hat{\mathbf{r}} ; \mathbf{k}) & =-\sum_{n=0}^{\infty} \frac{(i k)^{n+1}}{n !} \mathcal{H}_{n}(\hat{\mathbf{r}} ; \hat{\mathbf{k}}) \\
& =\sum_{n=0}^{\infty} \frac{(i k)^{n+1}}{n !} \sum_{\rho=0}^{n}\left(\begin{array}{l}
n \\
\rho
\end{array}\right)(-1)^{\rho+1} M_{n-\rho}^{\rho}(\hat{\mathbf{r}} ; \hat{\mathbf{k}}),
\end{aligned}
$$

where we recognize the low-frequency moments met in [3] and given by

$$
M_{l}^{m}(\hat{\mathbf{r}} ; \hat{\mathbf{k}})=\frac{1}{4 \pi} \int_{\partial D}\left(\hat{\mathbf{r}} \cdot \mathbf{r}^{\prime}\right)^{m} \frac{\partial \Phi_{l}}{\partial n^{\prime}}\left(\mathbf{r}^{\prime} ; \hat{\mathbf{k}}\right) d s\left(\mathbf{r}^{\prime}\right), \quad \hat{\mathbf{r}}, \hat{\mathbf{k}} \in S^{2} .
$$

We notice here that the dependence of the moments on the arguments is simplified in the cases (i) $M_{0}^{m}(\hat{\mathbf{r}} ; \hat{\mathbf{k}})=M_{0}^{m}(\hat{\mathbf{r}})$ (the zeroth-order approximation does not depend on the excitation direction) and (ii) $M_{l}^{0}(\hat{\mathbf{r}} ; \hat{\mathbf{k}})=M_{l}^{0}(\hat{\mathbf{k}})$ (the observation point is irrelevant).

The direct scattering problem in low frequencies consists of the solution of the abovementioned sequence of potential problems leading to the determination of the lowfrequency components $\Phi_{n}(\mathbf{r} ; \hat{\mathbf{k}})$, whose knowledge is necessary for the acquisition of the far field $f_{\infty}(\hat{\mathbf{r}} ; \mathbf{k})$ via the representation (9).

The inverse scattering problem is based on expansion (9) again, but in the opposite direction. A set of measurements of the far-field pattern $f_{\infty}(\hat{\mathbf{r}} ; \mathbf{k})$ for several possible observations (vectors $\hat{\mathbf{r}}$ ) and excitations (wave numbers $k$ and propagation directions $\hat{\mathbf{k}}$ ) is now given and this information must be decoded appropriately so that the surface $\partial D$ of the scatterer to be determined or at least suitably approximated. This task is characterized by several theoretical and practical issues as the existence and uniqueness of the solution, the stability of the reconstruction schemes and the examination of the influence of the qualitative and quantitative characteristics of the data (i.e., implication of noise in data, limiting aperture case, etc.) on the inverse problem solvability. 
3. The determination of the far field low-frequency components. In this section we present a stable process to obtain the low-frequency components $\mathcal{H}_{n}(\hat{\mathbf{r}} ; \hat{\mathbf{k}})$ from measurements of the far-field pattern. However this deduction must take into consideration the involvement of the underlying measurement noise and in general must confront the important issue of stabilization with respect to measurement inaccuracies.

The investigation of the reliable mining of the quantities $\mathcal{H}_{n}(\hat{\mathbf{r}} ; \hat{\mathbf{k}})$ from measurements is a multi-parameter problem and constitutes the aim of this section. The first step is to characterize fully the class of the admissible far-field patterns. Even if measurements are noisy, it is necessary to know exactly the nature of the "clear" data. This issue has to do with the concept of the characterization of the far-field pattern. Special effort has been devoted to the establishment of the necessary and sufficient conditions for a function defined on the unit sphere to stand as a radiation pattern $([11,[12])$. In acoustics then, it is well known that it is sufficient and necessary for a radiation pattern to constitute the restriction on the unit sphere $S^{2}$ of an entire analytic function whose $L^{2}$-norm on a surface of radius $R$ is of exponential type $O\left(e^{2 k C R}\right)$. The geometrical meaning of $C$ is just the radius of the sphere circumscribing the scatterer $D$. Denoting this set of radiation patterns as $\mathcal{P}\left(S^{2}\right)$, it is clear that $\mathcal{P}\left(S^{2}\right) \subset L^{2}\left(S^{2}\right)$, while $\left.\overline{\mathcal{P}\left(S^{2}\right)}\right|_{L^{2}}=L^{2}\left(S^{2}\right)$ for almost all wavenumbers $k$. The last assertion stems for the fact that $\mathcal{P}\left(S^{2}\right)$ includes the range of the well-known far-field operator [13], which is dense in the case that $k^{2}$ is not a Dirichlet eigenvalue of the negative Laplacian operator in $D$. To make clear the dependence on the wave number, we symbolize the set of admissible radiation patterns as $\mathcal{P}\left(S^{2} ; \mathbf{k}\right)$ and mention that this set includes functions analytic on $\hat{\mathbf{k}} \in S^{2}$, as well as on the wave number $k$. Moreover the members of $\mathcal{P}\left(S^{2} ; \mathbf{k}\right)$ constitute power series of $k$ in the Rayleigh region [8]. The set of square integrable functions depending parametrically on $\mathbf{k}$ is denoted as $L\left(S^{2} ; \mathbf{k}\right)$.

Let us denote the set including all possible polarizations and wave numbers in the low-frequency region as

$$
\mathcal{Q}=\mathcal{Q}\left(S^{2}\right)=\left\{\mathbf{k} \in R^{3}, k \in\left[0, k_{0}\right] ; \hat{\mathbf{k}} \in S^{2}\right\},
$$

where $k_{0}$ is selected as a close estimation of the upper bound of the Rayleigh region. The set of square integrable functions on the unit sphere $S^{2}$ (with respect to the orientation vector $\hat{\mathbf{r}})$ and on the phase space $\mathcal{Q}\left(S^{2}\right)$ is the Hilbert space $H_{1}=L^{2}\left(S^{2} ; Q\right)$. More precisely, $H_{1}$ is considered as the direct sum of Hilbert spaces

$$
\bigoplus_{\mathbf{k} \in \mathcal{Q}\left(S^{2}\right)} L^{2}\left(S^{2} ; \mathbf{k}\right)
$$

consisting of the set of all indexed families $f=f_{\mathbf{k}}$ with

$$
\sum_{\mathbf{k} \in \mathcal{Q}\left(S^{2}\right)}\left\|f_{\mathbf{k}}\right\|_{L^{2}}^{2}<\infty
$$

and endowed with the usual inner product

$$
\langle f, g\rangle=\left.\sum_{\mathbf{k} \in \mathcal{Q}\left(S^{2}\right)}\left\langle f_{\mathbf{k}}, g_{\mathbf{k}}\right\rangle\right|_{L^{2}} .
$$

$H_{1}$ is actually the space of all functions with domain $\mathcal{Q}\left(S^{2}\right)$ (where $\mathbf{k}$ belongs), such that $f_{\mathbf{k}}$ is an element of $L^{2}\left(S^{2}\right)$ for every possible $\mathbf{k}$. In addition we note that $f \in H_{1}$ iff 
$f=f(\hat{\mathbf{r}} ; \mathbf{k})$ with

$$
\int_{S^{2}} d \hat{\mathbf{r}} \int_{S^{2}} d \hat{\mathbf{k}} \int_{0}^{k_{0}} d k k^{2}|f(\hat{\mathbf{r}}, \mathbf{k})|^{2}<\infty .
$$

The family of all possible radiation patterns $\mathcal{P}\left(S^{2} ; \mathcal{Q}\right)$ is the set

$$
\bigoplus_{\mathbf{k} \in \mathcal{Q}\left(S^{2}\right)} \mathcal{P}\left(S^{2} ; \mathbf{k}\right)
$$

and is dense in $H_{1}$. A relevant definition holds for the notation $L^{2}\left(S^{2} ; S^{2}\right)$, where the phase space dependence concerns all possible orientations $\hat{\mathbf{k}} \in S^{2}$ of the incident field. We denote as $H_{2}$ the Hilbert space considered as the direct sum $\bigoplus_{n \in N \cup\{0\}} X_{n}$ (with $X_{n}=L^{2}\left(S^{2}, S^{2}\right)$ for every $\left.n\right)$, endowed with the weighted inner product

$$
\langle f, g\rangle=\left.\sum_{n=0}^{\infty} \frac{k_{0}^{2 n+2}}{(n !)^{2}}\left\langle f_{n}, g_{n}\right\rangle\right|_{L^{2}\left(S^{2}\right) \times L^{2}\left(S^{2}\right)} .
$$

Let us consider the operator $L: \mathcal{P}\left(S^{2} ; Q\right) \subseteq H_{1} \rightarrow H_{2}$ defined as

$$
L\left(f_{\infty}\right)=\left(\mathcal{H}_{0}, \mathcal{H}_{1}, \ldots, \mathcal{H}_{n}, \ldots\right) .
$$

In the case that we have restricted data in the directions forming the set $A\left(\subset S^{2}\right)$ and restricted excitation directions $\left(B \subset S^{2}\right)$, then the operator $L$ acts on

$$
\mathcal{P}(A ; \mathcal{Q}(B)) \subseteq H_{1}(A, B)=L^{2}(A ; \mathcal{Q}(B))
$$

and takes values in

$$
H_{2}(A, B)=\prod_{n \in N} X_{n}(A, B)
$$

with $X_{n}(A, B)=L^{2}(A, B)$. Furthermore if measurements refer to a proper (usually finite) subset of wavenumbers $\left(k \in I \subset\left[0, k_{0}\right]\right)$, then the operator $L_{I}$ arises by mapping $\mathcal{P}\left(A ; \mathcal{Q}_{I}(B)\right)$ into $H_{2}(A, B)$ where

$$
\mathcal{Q}_{I}(B)=\left\{\mathbf{k} \in R^{3}, k \in I \subset\left[0, k_{0}\right] ; \hat{\mathbf{k}} \in B\right\} .
$$

An important case is when the sets $A$ and $B$ are finite containing specific orientations. Then we denote $A_{N}=\left\{\hat{\mathbf{r}}_{i}, i=1,2, \ldots, N\right\}$ and $B_{M}=\left\{\hat{\mathbf{k}}_{i}, i=1,2, \ldots, M\right\}$ where we pay attention to the finite dimensionality $M(N)$ of the parametric space $B$ (of the set of measurement orientations $A$ ).

In practice we are usually given a sequence of measurements $f_{\infty}^{\delta} \in H_{1}(A, B)$, approximating the far-field pattern $f_{\infty} \in \mathcal{P}(A ; \mathcal{Q}(B))$, collected at observation directions in $A$, produced by excitations in $B$ and corresponding to concrete wavenumbers in $\left[0, k_{0}\right]$. The error in measurements is expressed through the level $\delta$, while we make the basic assumption that the signal to noise ratio is strictly bounded above one. We assume then that for some fixed $\tau>1$,

$$
\left\|f_{\infty}^{\delta}-f_{\infty}\right\| \leq \delta<\frac{1}{\tau}\left\|f_{\infty}^{\delta}\right\| .
$$

The aim of this section is to suggest a technique mining appropriately good estimations of the low-frequency components $\mathcal{H}_{n}, n=1,2,3, \ldots$ starting from the knowledge of $f_{\infty}^{\delta}$. To that purpose, we follow the basic ideas of [14, where efficient finite-dimensional 
stabilization techniques are developed to exploit suitably the Tikhonov regularization methodology. Let us consider again the operator

$$
L: \mathcal{P}(A ; Q(B)) \subseteq H_{1}(A, B) \rightarrow H_{2}(A, B)
$$

We construct an expanded chain of finite-dimensional spaces $V_{1} \subseteq V_{2} \subseteq \ldots \subseteq \mathcal{D}(L)$ such that $H_{1}(A, B) \subseteq \overline{\bigcup_{i=1}^{\infty} V_{i}}$. In the case that the spaces $A, B$ are finite dimensional, then $f \in V_{n}$ iff there exist constants $c_{n, l}^{i j}$ such that

$$
f=f\left(\hat{\mathbf{r}}_{i} ; k \hat{\mathbf{k}}_{j}\right)=\sum_{l=0}^{n} c_{n, l}^{i j} k^{l},
$$

for $i=1,2, \ldots, N, j=1,2, \ldots, M$ and $k \in\left[0, k_{0}\right]$. In the general case when $A, B$ are portions of the unit sphere of infinite cardinality, then

$$
V_{n}=\left\{f \in H_{1}(A, B): f(\hat{\mathbf{r}} ; \mathbf{k})=\sum_{l=0}^{n} f_{l}^{m(n)}(\hat{\mathbf{r}} ; \hat{\mathbf{k}}) k^{l}\right\}
$$

where $m(n)$ is an integer depending on the index $n(m(n) \stackrel{n \rightarrow \infty}{\longrightarrow} \infty)$ and $f_{l}^{m(n)}$ belongs to the space $W_{m(n)} \subset L^{2}\left(S^{2}\right) \times L^{2}\left(S^{2}\right)$, consisting of all the products of spherical harmonics $Y_{n^{\prime}}^{m^{\prime}}(\hat{\mathbf{r}}) Y_{n^{\prime \prime}}^{m^{\prime \prime}}(\hat{\mathbf{k}})$ of order $n^{\prime}, n^{\prime \prime}$ less than $m(n)$.

We consider the functional $\Phi_{\alpha}\left(\cdot ; f_{\infty}^{\delta}\right)$, defined on $\mathcal{D}(L)$ as follows:

$$
\Phi_{\alpha}\left(z ; f_{\infty}^{\delta}\right)=\left\|z-f_{\infty}^{\delta}\right\|^{2}+\alpha\|L z\|^{2} .
$$

We denote by $f_{n, \alpha}^{\delta}$ the finite-dimensional approximation in $V_{n}$ defined by

$$
f_{n, \alpha}^{\delta}=\operatorname{argmin}_{z \in V_{n}} \Phi_{\alpha}\left(z ; f_{\infty}^{\delta}\right) .
$$

We consider the projection operator $P_{n}: H_{1}(A, B) \rightarrow V_{n}$. It is easily verified [14] that

$$
f_{n, \alpha}^{\delta}=\left(I+\alpha L_{n}^{\star} L_{n}\right)^{-1} P_{n} f_{\infty}^{\delta},
$$

where $L_{n}=\left.L\right|_{V_{n}}$ is the restriction of $L$ to $V_{n}$. In order to handle the relationship between the approximate and the exact solutions, we need (consult again [14]) the validity of the convergence of the projection method (i.e., $L P_{n} f_{\infty} \rightarrow L f_{\infty}$, as $n \rightarrow \infty$, for every $\left.f_{\infty} \in \mathcal{D}(L)\right)$ or the weaker condition that $\lim _{n \rightarrow \infty}\left\|L P_{n} f_{\infty}\right\| \leq\left\|L f_{\infty}\right\|$. Both relations are easily proved due to the essence of the specific projection, consisting of keeping the polynomial part (of order $n$ ) of the analytic member (w.r.t $k$ ) of $\mathcal{D}(L)$. This step is necessary and sufficient for the establishment of the following convergence theorem.

ThEOREM 1. Suppose that $f_{\infty}$ belongs to $\mathcal{D}(L)=\mathcal{P}(A ; Q(B)) \subseteq H_{1}(A, B)=L^{2}(A ; Q(B))$. Assume that the measurement $f_{\infty}^{\delta}$ is provided where the error level $\delta(>0)$ is such that the relation (15) is satisfied. Then

(i) There exists $N_{\delta}$ such that

$$
\begin{aligned}
& \left\|L P_{n} f_{\infty}\right\|^{2} \leq \delta+\left\|L f_{\infty}\right\|^{2}, \\
& \left\|\left(I-P_{n}\right) f_{\infty}^{\delta}\right\| \leq \delta \\
& \tau \delta<\left\|P_{n} f_{\infty}^{\delta}\right\|
\end{aligned}
$$

for all $n \geq N_{\delta}$. 
(ii) Let $n(\delta)>N_{\delta}$. The function $d(\alpha)=\left\|f_{n, \alpha}^{\delta}-P_{n} f_{\infty}^{\delta}\right\|$ is continuous, strictly increasing, tends to 0 as $\alpha \rightarrow 0^{+}$and there exists a unique $\alpha=\alpha(n(\delta), \delta)$ such that

$$
\left\|f_{n, \alpha}^{\delta}-P_{n} f_{\infty}^{\delta}\right\|=\tau \delta
$$

(iii) For the above (ii) value of the stabilization parameter $\alpha$, we have

$$
f_{n, \alpha}^{\delta} \rightarrow f_{\infty} \quad \text { and } \quad L f_{n, \alpha}^{\delta} \rightarrow L f_{\infty} \quad \text { as } \delta \rightarrow 0 .
$$

Proof. The results of the theorem are reformulated issues of the paragraph 5.2.1 of 14, adapted to the current situation.

Part (iii) of the above theorem assures the convergence of the approximated estimations $f_{n, \alpha}^{\delta}$ of the far field (based on the noisy data $f_{\infty}^{\delta}$ ) to the exact data $f_{\infty}$ and (under the application of $L)$ to the exact far-field low-frequency components $\left\{\mathcal{H}_{n} ; n=0,1,2, \ldots\right\}$.

The implementation of the method suggested by the preceding theorem is well realized when we consider the case of finite observation and excitation sets $A$ and $B$. In that case the situation is getting simpler and some of the previous steps degenerate to trivial processes. As a clarifying example, let us consider the case of just one specific excitation $\hat{\mathbf{k}}_{0}$ and only one observation direction $\hat{\mathbf{r}}_{0}$, in which we would like to estimate the lowfrequency components. The elements of $V_{n}$ obtain the simple form $z=\sum_{l=0}^{n} c_{n, l} k^{l}$. The functional $\Phi_{\alpha}\left(z ; f_{\infty}^{\delta}\right)$ defined on $V_{n}$ obtains the form

$$
\Phi_{\alpha}\left(z ; f_{\infty}^{\delta}\right)=\int_{0}^{k_{0}} d k k^{2}\left|\sum_{l=0}^{n} c_{n, l} k^{l}-f^{\delta}(k)\right|^{2}+\alpha \sum_{l=0}^{n} \frac{k_{0}^{2 l+2}}{(n !)^{2}}\left|c_{n, l}\right|^{2},
$$

where $f^{\delta}(k)=f_{\infty}^{\delta}\left(\hat{\mathbf{r}}_{0} ; k \hat{\mathbf{k}}_{0}\right)$.

Minimizing over the coefficients $c_{n, l}$, we obtain the linear system

$$
\begin{array}{r}
\left(\alpha \frac{k_{0}^{2 l+2}}{(n !)^{2}}+\frac{k_{0}^{2 l+3}}{2 l+3}\right) c_{n, l}^{\alpha}+\sum_{l^{\prime} \neq l} \frac{k_{0}^{l+l^{\prime}+3}}{l+l^{\prime}+3} c_{n, l^{\prime}}^{\alpha}=\int_{0}^{k_{0}} d k k^{l+2} f^{\delta}(k), \\
\quad l=0,1,2, \ldots, n .
\end{array}
$$

The parameter $\alpha$ has of course wave-number dimensions and is selected, according to (20), such that $\int_{0}^{k_{0}} d k k^{2}\left|\sum_{l=0}^{n} c_{n, l}^{\alpha} k^{l}-f^{\delta}(k)\right|^{2}=\tau \delta$.

Usually, we do not dispose of $f^{\delta}(k)$ for all values of $k \in\left[0, k_{0}\right]$ but for some discrete frequencies in this interval. This leads to a consequent discretization of the integral appearing at the r.h.s. of (23) and a more general appropriate discrete modification of the whole system (23). The main result (iii) of the theorem of this section assures the convergence $L f_{n, \alpha}^{\delta} \rightarrow L f_{\infty}$, i.e. $\left(c_{n, 0}^{\alpha}, \ldots, c_{n, n}^{\alpha}, 0,0, \ldots\right) \rightarrow\left(\mathcal{H}_{0}, \ldots, \mathcal{H}_{i}, \mathcal{H}_{i+1}, \ldots\right)$ as $\delta \rightarrow 0$, where in this simple case, $\mathcal{H}_{i}$ stands for the single value $\mathcal{H}_{i}\left(\hat{\mathbf{r}}_{0}, \hat{\mathbf{k}}_{0}\right)$ for every $i=1,2,3, \ldots$.

4. Investigation of the low-frequency moments. The old version of the moments method aimed at the determination of smooth scatterers, would the moments $M_{0}^{m}(\hat{\mathbf{r}} ; \hat{\mathbf{k}})$ (pertaining exclusively to the capacity (zeroth-order) potential) be given, without consideration to the process of obtaining these moments from the real measurements of the problem. Indeed, in these early days, the fundamental theoretical interest of the authors stemmed primitively from the well-known theoretical work of Minkowski, assuring the 
ability to reconstruct a surface after the curvature is determined everywhere on the surface. Of course the determination of the curvature is a nonlinear difficult problem but in the case under discussion the authors had conceived that the Rayleigh low-frequency moments had some very fruitful properties: They constituted the structural elements of the known far-field pattern and on the other hand transferred the curvature of the scatterer in a concealed but very particular integral form. So the curvature was brought into the light of exploration via the carriers of the moments. In fact the curvature was involved in the surface measure induced by the capacity potential. The amazing fact was that the curvature remained concealed until the end, but its particular involvement in the polar generalized moments (produced in a methodological though complicated manner from the original ones) was the basic ingredient for the determination of the polar representation [3] of the scatterer's surface. In other words the moments method detours the determination of the curvature but exploits its indirect presence in the integral representations to determine the polar form of the surface itself. This result was an affirmative response to the theoretical challenge originated from the concept of Minkowski and no effort was then devoted to further investigation of the corresponding inverse problem.

The approach of the present work is characterized in principle by the motive to define the path originated from the far-field information, passing from the treatment of the moments and leading finally to the solution of the inverse scattering problem. In this section, we simply mention that the starting point of the process is the low-frequency expansion (9) of the far-field pattern. Following the Tichonov regularized scheme presented in the previous section to ensure the stabilization in the measurement process, we are in a position to obtain the far-field low-frequency components

$$
\mathcal{H}_{n}(\hat{\mathbf{r}} ; \hat{\mathbf{k}})=\sum_{\rho=0}^{n}\left(\begin{array}{l}
n \\
\rho
\end{array}\right)(-1)^{\rho} M_{n-\rho}^{\rho}(\hat{\mathbf{r}} ; \hat{\mathbf{k}}), n=0,1,2, \ldots
$$

after exploiting the knowledge of the far-field pattern for several wave numbers $k$ in the low-frequency region. So we are able to determine the functions $M_{0}^{0}(\hat{\mathbf{r}} ; \hat{\mathbf{k}}), M_{1}^{0}(\hat{\mathbf{r}} ; \hat{\mathbf{k}})-$ $M_{0}^{1}(\hat{\mathbf{r}} ; \hat{\mathbf{k}}), M_{2}^{0}(\hat{\mathbf{r}} ; \hat{\mathbf{k}})-2 M_{1}^{1}(\hat{\mathbf{r}} ; \hat{\mathbf{k}})+M_{0}^{2}(\hat{\mathbf{r}} ; \hat{\mathbf{k}})$, etc., for all observation and excitation directions.

It is important to describe a systematic manner to define and then to mine a useful subset along the moments $M_{l}^{n}(\hat{\mathbf{r}} ; \hat{\mathbf{k}}), l=0,1,2, \ldots, n=0,1,2, \ldots$ (or at least some useful generalized moments stemming from them) by exploiting the determined functions $\mathcal{H}_{l}(\hat{\mathbf{r}} ; \hat{\mathbf{k}})$. The mechanism suggested in [3] was based on expanding the functions $\mathcal{H}_{l}(\hat{\mathbf{r}} ; \hat{\mathbf{k}})$, $l=0,1,2, \ldots$, in terms of spherical harmonics w.r.t. the observation vector $\hat{\mathbf{r}}$ and then using the orthogonality properties of these functions to separate the moments. This is a very useful, from a theoretical point of view, method, which is going to be exploited again in the present framework. However this approach will not be adopted in the final step of the determination of the necessary moments, since it has the drawback that it requires using the entire information for all possible observation directions. This is generally undesirable and is not aligned with the reasonable rule that at least for polynomial scatterers (or scatterers approximated suitably by polynomial surfaces), the lower the degree of the surface, the smaller is the number of the needed observation directions. We 
are going then to develop a process to obtain all the necessary, in this work, moments, using the optimal number of observation directions.

The path between the two aforementioned approaches will immediately be apparent through the next ideas. The forthcoming first result reveals the kind of the generalized moments (deduced by the zeroth-order low-frequency moments) that can be produced, at least theoretically, from the far-field components.

Proposition 2. For every $n=0,1,2, \ldots$, the generalized zeroth-order moments $\mathcal{M}_{0}^{(n, m)}$ given by the integrals

$$
\mathcal{M}_{0}^{(n, m)}=\frac{1}{4 \pi} \int_{\partial D} Y_{n}^{m}(\hat{\mathbf{r}}) r^{n} \frac{\partial \Phi_{0}}{\partial n}(\mathbf{r}) d s(\mathbf{r}), \quad|m| \leq n,
$$

where $Y_{n}^{m}(\hat{\mathbf{r}})$ stand for the well-known spherical harmonics, are reconstructible once the far-field low-frequency component $\mathcal{H}_{n}(\hat{\mathbf{r}} ; \hat{\mathbf{k}})$ is given.

Proof. We notice first that the scatterer's surface is star-shaped and so $\mathbf{r}=r(\hat{\mathbf{r}}) \hat{\mathbf{r}}$ sweeps $\partial D$ as $\hat{\mathbf{r}}$ runs over $S^{2}$ with a well-defined smooth function $r(\hat{\mathbf{r}})$.

The proof is simple and is based on analyzing the powers $\left(\hat{\mathbf{r}} \cdot \mathbf{r}^{\prime}\right)^{q}$, appearing in the kernels of the moments participating in the form of the component $\mathcal{H}_{n}(\hat{\mathbf{r}} ; \hat{\mathbf{k}})$, first in terms of Legendre functions through the well-known formulae [15]

$$
\begin{aligned}
\left(\hat{\mathbf{r}} \cdot \hat{\mathbf{r}}^{\prime}\right)^{2 l}= & \frac{1}{2 l+1} P_{0}\left(\hat{\mathbf{r}} \cdot \hat{\mathbf{r}}^{\prime}\right)+\frac{5 \cdot 2 l}{(2 l+1)(2 l+3)} P_{2}\left(\hat{\mathbf{r}} \cdot \hat{\mathbf{r}}^{\prime}\right)+\ldots \\
& +\frac{(4 l+1) 2 l(2 l-2) \ldots 2}{(2 l+1)(2 l+3) \ldots(4 l+1)} P_{2 l}\left(\hat{\mathbf{r}} \cdot \hat{\mathbf{r}}^{\prime}\right), l=0,1,2, \ldots \\
\left(\hat{\mathbf{r}} \cdot \hat{\mathbf{r}}^{\prime}\right)^{2 l+1}= & \frac{3}{2 l+3} P_{1}\left(\hat{\mathbf{r}} \cdot \hat{\mathbf{r}}^{\prime}\right)+\frac{7 \cdot 2 l}{(2 l+3)(2 l+5)} P_{3}\left(\hat{\mathbf{r}} \cdot \hat{\mathbf{r}}^{\prime}\right)+\ldots \\
& +\frac{(4 l+3) 2 l(2 l-2) \ldots 2}{(2 l+3)(2 l+5) \ldots(4 l+3)} P_{2 l+1}\left(\hat{\mathbf{r}} \cdot \hat{\mathbf{r}}^{\prime}\right), l=0,1,2, \ldots
\end{aligned}
$$

and then in terms of spherical harmonics via the relations

$$
P_{n}\left(\hat{\mathbf{r}} \cdot \hat{\mathbf{r}}^{\prime}\right)=\sum_{m=-n}^{n} \frac{(n-|m|) !}{(n+|m|) !} \overline{Y_{n}^{m}(\hat{\mathbf{r}})} Y_{n}^{m}\left(\hat{\mathbf{r}}^{\prime}\right) .
$$

Then $\mathcal{H}_{n}(\hat{\mathbf{r}} ; \hat{\mathbf{k}})$ obtains a polar form where the terms corresponding to spherical harmonics of the highest degree $n$ are provided exclusively by the moment $M_{0}^{n}(\hat{\mathbf{r}} ; \hat{\mathbf{k}})$. More precisely, we have

$$
\begin{array}{r}
\mathcal{H}_{n}(\hat{\mathbf{r}} ; \hat{\mathbf{k}})=(-1)^{n} \frac{(2 n+1) \cdot 2\left[\frac{n}{2}\right] \cdot\left(2\left[\frac{n}{2}\right]-2\right) \cdots 2}{\left(2 n+1-2\left[\frac{n}{2}\right]\right) \cdot\left(2 n+3-2\left[\frac{n}{2}\right]\right) \cdots(2 n+1)} \\
\times \sum_{m=-n}^{n} \frac{(n-|m|) !}{(n+|m|) !} \frac{}{Y_{n}^{m}(\hat{\mathbf{r}})} \mathcal{M}_{0}^{(n, m)}+(\text { sph. harmonics of order }<n),
\end{array}
$$

which immediately guarantees the ability to recover $\mathcal{M}_{0}^{(n, m)}$ from the far-field lowfrequency components.

Referring to (28), we notice that the terms corresponding to spherical harmonics of order $n^{\prime}<n-1$ involve the zeroth-order generalized moments $\mathcal{M}_{0}^{\left(n^{\prime}, m, n\right)}=$ 
$\frac{1}{4 \pi} \int_{\partial D} Y_{n^{\prime}}^{m}(\hat{\mathbf{r}}) r^{n} \frac{\partial \Phi_{0}}{\partial n}(\mathbf{r}) d s(\mathbf{r}),|m| \leq n^{\prime}$ together with generalized moments stemming from higher-order low-frequency approximations $\Phi_{n}$. As an example, the moment $\mathcal{M}_{0}^{(n-2, m, n)}$ is combined with $\mathcal{M}_{2}^{(n-2, m)}$ to compose the coefficient of $\overline{Y_{n-2}^{m}(\hat{\mathbf{r}})}$ in (28).

REMARK 3. It is interesting to mention that the privileged independent generalized moments $\mathcal{M}_{0}^{(n, m)}$ constitute integrals imitating "inner products" of $\frac{\partial \Phi_{0}}{\partial n}(\mathbf{r})$ with all the harmonic functions $r^{n} Y_{n}^{m}(\hat{\mathbf{r}})$ of degree $n$ (as $m$ runs over all its possible values), while the integrands in the remaining generalized moments $\mathcal{M}_{0}^{\left(n^{\prime}, m, n\right)}$ have lost this behavior. In addition $\mathcal{M}_{0}^{(n, m)}$ can be transformed easily to "inner products" with all the possible harmonics transferred in cartesian form, giving place to the moments $\widetilde{\mathcal{M}}_{0}^{(n, j)}$, $j=1,2, \ldots, 2 n+1$. As an example the set of $\mathcal{M}_{0}^{(2, m)},|m| \leq 2$ is equivalent with the set of moments

$$
\widetilde{\mathcal{M}}_{0}^{(2, j)}=\frac{1}{4 \pi} \int_{\partial D} h_{2, j}(\mathbf{r}) \frac{\partial \Phi_{0}}{\partial n}(\mathbf{r}) d s(\mathbf{r}), \quad j=1,2, \ldots, 5,
$$

where $h_{2, j}(\mathbf{r}) \in\left\{x^{2}-y^{2}, x y, x z, y z, x^{2}-z^{2}\right\}$. It will be clear in the inversion algorithm [6] that "tilda"-type moments emerge in a natural way and form the structural elements of the inversion scheme.

As mentioned before, proving the ability to determine $\mathcal{M}_{0}^{(n, m)}$ is only the beginning. It is necessary to obtain these moments in an efficient manner for the solution of the inverse problem. The next two lemmas answer partially the question about the reasonable mining of the generalized moments from measurements.

Lemma 4. The moments $M_{0}^{0}, M_{0}^{1}(\hat{\mathbf{r}})$ and $M_{1}^{0}(\hat{\mathbf{k}})$ are directly deducible from measurements.

Proof. It is clear that

$$
\begin{aligned}
M_{0}^{0} & =\mathcal{H}_{0}, \\
M_{0}^{1}(\hat{\mathbf{r}}) & =\frac{1}{2}\left[\mathcal{H}_{1}(-\hat{\mathbf{r}} ; \hat{\mathbf{k}})-\mathcal{H}_{1}(\hat{\mathbf{r}} ; \hat{\mathbf{k}})\right], \\
M_{1}^{0}(\hat{\mathbf{k}}) & =\frac{1}{2}\left[\mathcal{H}_{1}(\hat{\mathbf{r}} ; \hat{\mathbf{k}})+\mathcal{H}_{1}(-\hat{\mathbf{r}} ; \hat{\mathbf{k}})\right] .
\end{aligned}
$$

Observe that $\widetilde{\mathcal{M}}_{0}^{(1,1)}\left(=\frac{1}{4 \pi} \int_{\partial D} x \frac{\partial \Phi_{0}}{\partial n}(\mathbf{r}) d s(\mathbf{r})\right)=M_{0}^{1}(\hat{\mathbf{x}}), \widetilde{\mathcal{M}}_{0}^{(1,2)}=M_{0}^{1}(\hat{\mathbf{y}})$ and $\widetilde{\mathcal{M}}_{0}^{(1,3)}=$ $M_{0}^{1}(\hat{\mathbf{z}})$.

LEMma 5. For the determination of moments $\mathcal{M}_{0}^{(2, m)},|m| \leq 2$, or equivalently (according to Remark (3) for the construction of $\widetilde{\mathcal{M}}_{0}^{(2, j)}, j=1,2, \ldots, 5$, only 12 observation directions are needed.

Proof. It is clear from the definition of the far-field low-frequency components (24) that

$$
\mathcal{H}_{2}^{s y m}(\hat{\mathbf{r}} ; \hat{\mathbf{k}}) \stackrel{\operatorname{def}}{=} \frac{1}{2}\left[\mathcal{H}_{2}(\hat{\mathbf{r}} ; \hat{\mathbf{k}})+\mathcal{H}_{2}(-\hat{\mathbf{r}} ; \hat{\mathbf{k}})\right]=M_{0}^{2}(\hat{\mathbf{r}})+M_{2}^{0}(\hat{\mathbf{k}})
$$

Consequently

$$
\frac{1}{4 \pi} \int_{\partial D}\left(x^{2}-y^{2}\right) \frac{\partial \Phi_{0}}{\partial n}(\mathbf{r}) d s(\mathbf{r})=\mathcal{H}_{2}^{s y m}(\hat{\mathbf{x}} ; \hat{\mathbf{k}})-\mathcal{H}_{2}^{s y m}(\hat{\mathbf{y}} ; \hat{\mathbf{k}}),
$$




$$
\begin{aligned}
\frac{1}{4 \pi} \int_{\partial D}\left(x^{2}-z^{2}\right) \frac{\partial \Phi_{0}}{\partial n}(\mathbf{r}) d s(\mathbf{r}) & =\mathcal{H}_{2}^{s y m}(\hat{\mathbf{x}} ; \hat{\mathbf{k}})-\mathcal{H}_{2}^{s y m}(\hat{\mathbf{z}} ; \hat{\mathbf{k}}), \\
\frac{1}{4 \pi} \int_{\partial D} x y \frac{\partial \Phi_{0}}{\partial n}(\mathbf{r}) d s(\mathbf{r}) & =\mathcal{H}_{2}^{s y m}\left(\frac{1}{\sqrt{2}}(\hat{\mathbf{x}}+\hat{\mathbf{y}}) ; \hat{\mathbf{k}}\right)-\frac{1}{2} \mathcal{H}_{2}^{s y m}(\hat{\mathbf{x}} ; \hat{\mathbf{k}}) \\
& -\frac{1}{2} \mathcal{H}_{2}^{s y m}(\hat{\mathbf{y}} ; \hat{\mathbf{k}}), \\
\frac{1}{4 \pi} \int_{\partial D} x z \frac{\partial \Phi_{0}}{\partial n}(\mathbf{r}) d s(\mathbf{r}) & =\mathcal{H}_{2}^{s y m}\left(\frac{1}{\sqrt{2}}(\hat{\mathbf{x}}+\hat{\mathbf{z}}) ; \hat{\mathbf{k}}\right)-\frac{1}{2} \mathcal{H}_{2}^{s y m}(\hat{\mathbf{x}} ; \hat{\mathbf{k}}) \\
& -\frac{1}{2} \mathcal{H}_{2}^{s y m}(\hat{\mathbf{z}} ; \hat{\mathbf{k}}), \\
\frac{1}{4 \pi} \int_{\partial D} y z \frac{\partial \Phi_{0}}{\partial n}(\mathbf{r}) d s(\mathbf{r}) & =\mathcal{H}_{2}^{s y m}\left(\frac{1}{\sqrt{2}}(\hat{\mathbf{y}}+\hat{\mathbf{z}}) ; \hat{\mathbf{k}}\right)-\frac{1}{2} \mathcal{H}_{2}^{s y m}(\hat{\mathbf{y}} ; \hat{\mathbf{k}}) \\
& -\frac{1}{2} \mathcal{H}_{2}^{s y m}(\hat{\mathbf{z}} ; \hat{\mathbf{k}}) .
\end{aligned}
$$

The same technique can be applied to reconstruct generalized moments of higher degree. The next result is indicative for the development of our approach.

Proposition 6. The moments $\widetilde{\mathcal{M}}_{0}^{(3, j)}, j=1,2, \ldots, 7$ are deducible from measurements.

Proof. The starting point is the representation (24) for $n=3$ :

$$
\mathcal{H}_{3}(\hat{\mathbf{r}} ; \hat{\mathbf{k}})=M_{3}^{0}(\hat{\mathbf{k}})-3 M_{2}^{1}(\hat{\mathbf{r}} ; \hat{\mathbf{k}})+3 M_{1}^{2}(\hat{\mathbf{r}} ; \hat{\mathbf{k}})-M_{0}^{3}(\hat{\mathbf{r}}) .
$$

We infer easily that

$$
3 M_{2}^{1}(\hat{\mathbf{r}} ; \hat{\mathbf{k}})+M_{0}^{3}(\hat{\mathbf{r}})=\frac{1}{2}\left[\mathcal{H}_{3}(-\hat{\mathbf{r}} ; \hat{\mathbf{k}})-\mathcal{H}_{3}(\hat{\mathbf{r}} ; \hat{\mathbf{k}})\right] \stackrel{\text { def }}{=}-\mathcal{H}_{3}^{a n t}(\hat{\mathbf{r}} ; \hat{\mathbf{k}}) .
$$

Expanding suitably the integrands in spherical harmonics, we find that

$$
\begin{array}{r}
\frac{3}{4 \pi} \int_{\partial D}\left(\hat{\mathbf{r}} \cdot \mathbf{r}^{\prime}\right) \frac{\partial \Phi_{2}}{\partial n^{\prime}}\left(\mathbf{r}^{\prime} ; \hat{\mathbf{k}}\right) d s\left(\mathbf{r}^{\prime}\right)+\frac{3}{20 \pi} \int_{\partial D}\left(\hat{\mathbf{r}} \cdot \mathbf{r}^{\prime}\right) r^{\prime 2} \frac{\partial \Phi_{0}}{\partial n^{\prime}}\left(\mathbf{r}^{\prime}\right) d s\left(\mathbf{r}^{\prime}\right) \\
+\frac{2}{5} \sum_{m=-3}^{3} \frac{(3-|m|) !}{(3+|m|) !} \overline{Y_{3}^{m}(\hat{\mathbf{r}})} \mathcal{M}_{0}^{(3, m)}=-\mathcal{H}_{3}^{a n t}(\hat{\mathbf{r}} ; \hat{\mathbf{k}}) .
\end{array}
$$

We apply (34) for three orientations forming an orthonormal basis $\hat{\mathbf{r}}_{i}, i=1,2,3 \in R^{3}$. Multiplying each relation with the corresponding unit vector $\hat{\mathbf{r}}_{i}$, adding over $i=1,2,3$ and using the well-known representation of the unit dyadic $\mathcal{I}=\sum_{i=1}^{3} \hat{\mathbf{r}}_{i} \hat{\mathbf{r}}_{i}$, we obtain

$$
\mathcal{B}_{1}(\hat{\mathbf{k}})+\frac{2}{5} \sum_{m=-3}^{3} \frac{(3-|m|) !}{(3+|m|) !} \sum_{i=1}^{3}\left[\hat{\mathbf{r}}_{i} \overline{Y_{3}^{m}\left(\hat{\mathbf{r}}_{i}\right)}\right] \mathcal{M}_{0}^{(3, m)}=-\sum_{i=1}^{3}\left[\hat{\mathbf{r}}_{i} \mathcal{H}_{3}^{a n t}\left(\hat{\mathbf{r}}_{i} ; \hat{\mathbf{k}}\right)\right],
$$

where

$$
\mathcal{B}_{1}(\hat{\mathbf{k}})=\frac{3}{4 \pi} \int_{\partial D} \mathbf{r}^{\prime} \frac{\partial \Phi_{2}}{\partial n^{\prime}}\left(\mathbf{r}^{\prime} ; \hat{\mathbf{k}}\right) d s\left(\mathbf{r}^{\prime}\right)+\frac{3}{20 \pi} \int_{\partial D} \mathbf{r}^{\prime} r^{\prime 2} \frac{\partial \Phi_{0}}{\partial n^{\prime}}\left(\mathbf{r}^{\prime}\right) d s\left(\mathbf{r}^{\prime}\right)
$$

depends only in the excitation orientation $\hat{\mathbf{k}}$.

We make two different selections for the orthonormal basis: (a) $(\hat{\mathbf{x}}, \hat{\mathbf{y}}, \hat{\mathbf{z}})$ and (b) $\left(\frac{1}{\sqrt{2}}(\hat{\mathbf{x}}+\hat{\mathbf{y}}), \frac{1}{\sqrt{2}}(\hat{\mathbf{x}}-\hat{\mathbf{y}}), \hat{\mathbf{z}}\right)$. 
In the first case, (35) provides, after extended manipulations, the following relation:

$$
\begin{aligned}
& \frac{5}{2} \mathcal{B}_{1}(\hat{\mathbf{k}})+\hat{\mathbf{z}} \mathcal{M}_{0}^{(3,0)}-3 \frac{2 !}{4 !}\left[\Re\left(\mathcal{M}_{0}^{(3,1)}\right) \hat{\mathbf{x}}+\Im\left(\mathcal{M}_{0}^{(3,1)}\right) \hat{\mathbf{y}}\right] \\
& +30 \frac{1}{6 !}\left[\Re\left(\mathcal{M}_{0}^{(3,3)}\right) \hat{\mathbf{x}}-\Im\left(\mathcal{M}_{0}^{(3,3)}\right) \hat{\mathbf{y}}\right]=-\frac{5}{2} \sum_{i=1}^{3} \hat{\mathbf{r}}_{i}^{(a)} \mathcal{H}_{3}^{a n t}\left(\hat{\mathbf{r}}_{i}^{(a)} ; \hat{\mathbf{k}}\right),
\end{aligned}
$$

where the superscript $(a)$ indicates the first choice of vectors and the real and imaginary parts of the moments have been merged.

Repeating the same analysis for selection $(b)$, we find that

$$
\begin{aligned}
& \frac{5}{2} \mathcal{B}_{1}(\hat{\mathbf{k}})+\hat{\mathbf{z}} \mathcal{M}_{0}^{(3,0)}-3 \frac{2 !}{4 !}\left[\Re\left(\mathcal{M}_{0}^{(3,1)}\right) \hat{\mathbf{x}}+\Im\left(\mathcal{M}_{0}^{(3,1)}\right) \hat{\mathbf{y}}\right] \\
& -30 \frac{1}{6 !}\left[\Re\left(\mathcal{M}_{0}^{(3,3)}\right) \hat{\mathbf{x}}-\Im\left(\mathcal{M}_{0}^{(3,3)}\right) \hat{\mathbf{y}}\right]=-\frac{5}{2} \sum_{i=1}^{3} \hat{\mathbf{r}}_{i}^{(b)} \mathcal{H}_{3}^{a n t}\left(\hat{\mathbf{r}}_{i}^{(b)} ; \hat{\mathbf{k}}\right) .
\end{aligned}
$$

Subtracting (37/38), we find

$$
\begin{aligned}
& \Re\left(\mathcal{M}_{0}^{(3,3)}\right) \hat{\mathbf{x}}-\Im\left(\mathcal{M}_{0}^{(3,3)}\right) \hat{\mathbf{y}}=30\left\{\frac{1}{\sqrt{2}}(\hat{\mathbf{x}}+\hat{\mathbf{y}}) \mathcal{H}_{3}^{a n t}\left(\frac{1}{\sqrt{2}}(\hat{\mathbf{x}}+\hat{\mathbf{y}}) ; \hat{\mathbf{k}}\right)\right. \\
& \left.+\frac{1}{\sqrt{2}}(\hat{\mathbf{x}}-\hat{\mathbf{y}}) \mathcal{H}_{3}^{a n t}\left(\frac{1}{\sqrt{2}}(\hat{\mathbf{x}}-\hat{\mathbf{y}}) ; \hat{\mathbf{k}}\right)-\hat{\mathbf{x}} \mathcal{H}_{3}^{a n t}(\hat{\mathbf{x}} ; \hat{\mathbf{k}})-\hat{\mathbf{y}} \mathcal{H}_{3}^{a n t}(\hat{\mathbf{y}} ; \hat{\mathbf{k}})\right\}
\end{aligned}
$$

Expressing the harmonic kernels of $\mathcal{M}_{0}^{(3,3)}$ in terms of cartesian coordinates and projecting relation (39) on the axes $\hat{\mathbf{x}}$ and $\hat{\mathbf{y}}$, we find respectively that

$$
\begin{aligned}
& \frac{1}{4 \pi} \int_{\partial D}\left(x^{3}-3 x y^{2}\right) \frac{\partial \Phi_{0}}{\partial n}(\mathbf{r}) d s(\mathbf{r})=\sqrt{2} \mathcal{H}_{3}^{a n t}\left(\frac{1}{\sqrt{2}}(\hat{\mathbf{x}}+\hat{\mathbf{y}}) ; \hat{\mathbf{k}}\right) \\
& +\sqrt{2} \mathcal{H}_{3}^{a n t}\left(\frac{1}{\sqrt{2}}(\hat{\mathbf{x}}-\hat{\mathbf{y}}) ; \hat{\mathbf{k}}\right)-2 \mathcal{H}_{3}^{a n t}(\hat{\mathbf{x}} ; \hat{\mathbf{k}}), \\
& \frac{1}{4 \pi} \int_{\partial D}\left(y^{3}-3 y x^{2}\right) \frac{\partial \Phi_{0}}{\partial n}(\mathbf{r}) d s(\mathbf{r})=\sqrt{2} \mathcal{H}_{3}^{a n t}\left(\frac{1}{\sqrt{2}}(\hat{\mathbf{x}}+\hat{\mathbf{y}}) ; \hat{\mathbf{k}}\right) \\
& -\sqrt{2} \mathcal{H}_{3}^{a n t}\left(\frac{1}{\sqrt{2}}(\hat{\mathbf{x}}-\hat{\mathbf{y}}) ; \hat{\mathbf{k}}\right)-2 \mathcal{H}_{3}^{a n t}(\hat{\mathbf{y}} ; \hat{\mathbf{k}}) .
\end{aligned}
$$

In a very symmetric manner we find that

$$
\begin{aligned}
& \frac{1}{4 \pi} \int_{\partial D}\left(y^{3}-3 y z^{2}\right) \frac{\partial \Phi_{0}}{\partial n}(\mathbf{r}) d s(\mathbf{r})=\sqrt{2} \mathcal{H}_{3}^{a n t}\left(\frac{1}{\sqrt{2}}(\hat{\mathbf{y}}+\hat{\mathbf{z}}) ; \hat{\mathbf{k}}\right) \\
& +\sqrt{2} \mathcal{H}_{3}^{a n t}\left(\frac{1}{\sqrt{2}}(\hat{\mathbf{y}}-\hat{\mathbf{z}}) ; \hat{\mathbf{k}}\right)-2 \mathcal{H}_{3}^{a n t}(\hat{\mathbf{y}} ; \hat{\mathbf{k}}) \\
& \frac{1}{4 \pi} \int_{\partial D}\left(z^{3}-3 z y^{2}\right) \frac{\partial \Phi_{0}}{\partial n}(\mathbf{r}) d s(\mathbf{r})=\sqrt{2} \mathcal{H}_{3}^{a n t}\left(\frac{1}{\sqrt{2}}(\hat{\mathbf{y}}+\hat{\mathbf{z}}) ; \hat{\mathbf{k}}\right) \\
& -\sqrt{2} \mathcal{H}_{3}^{a n t}\left(\frac{1}{\sqrt{2}}(\hat{\mathbf{y}}-\hat{\mathbf{z}}) ; \hat{\mathbf{k}}\right)-2 \mathcal{H}_{3}^{a n t}(\hat{\mathbf{z}} ; \hat{\mathbf{k}})
\end{aligned}
$$

and

$$
\frac{1}{4 \pi} \int_{\partial D}\left(z^{3}-3 z x^{2}\right) \frac{\partial \Phi_{0}}{\partial n}(\mathbf{r}) d s(\mathbf{r})=\sqrt{2} \mathcal{H}_{3}^{a n t}\left(\frac{1}{\sqrt{2}}(\hat{\mathbf{z}}+\hat{\mathbf{x}}) ; \hat{\mathbf{k}}\right)
$$




$$
\begin{aligned}
& +\sqrt{2} \mathcal{H}_{3}^{a n t}\left(\frac{1}{\sqrt{2}}(\hat{\mathbf{z}}-\hat{\mathbf{x}}) ; \hat{\mathbf{k}}\right)-2 \mathcal{H}_{3}^{a n t}(\hat{\mathbf{z}} ; \hat{\mathbf{k}}) \\
& \frac{1}{4 \pi} \int_{\partial D}\left(x^{3}-3 x z^{2}\right) \frac{\partial \Phi_{0}}{\partial n}(\mathbf{r}) d s(\mathbf{r})=\sqrt{2} \mathcal{H}_{3}^{a n t}\left(\frac{1}{\sqrt{2}}(\hat{\mathbf{z}}+\hat{\mathbf{x}}) ; \hat{\mathbf{k}}\right) \\
& -\sqrt{2} \mathcal{H}_{3}^{a n t}\left(\frac{1}{\sqrt{2}}(\hat{\mathbf{z}}-\hat{\mathbf{x}}) ; \hat{\mathbf{k}}\right)-2 \mathcal{H}_{3}^{a n t}(\hat{\mathbf{x}} ; \hat{\mathbf{k}}) .
\end{aligned}
$$

Although we followed a constructive systematic manner to produce the moments of third degree, it is very easy to verify the above relations, starting from the right-hand side, exploiting the form of $\mathcal{H}_{3}^{a n t}$ and doing simple algebra. In the same spirit, we can show that

$$
\begin{aligned}
& \frac{1}{4 \pi} \int_{\partial D} x y z \frac{\partial \Phi_{0}}{\partial n}(\mathbf{r}) d s(\mathbf{r})=\frac{\sqrt{3}}{4}\left\{\mathcal{H}_{3}^{a n t}\left(\frac{1}{\sqrt{3}}(\hat{\mathbf{x}}+\hat{\mathbf{y}}-\hat{\mathbf{z}}) ; \hat{\mathbf{k}}\right)\right. \\
& -\mathcal{H}_{3}^{a n t}\left(\frac{1}{\sqrt{3}}(\hat{\mathbf{x}}+\hat{\mathbf{y}}+\hat{\mathbf{z}}) ; \hat{\mathbf{k}}\right)+\mathcal{H}_{3}^{a n t}\left(\frac{1}{\sqrt{3}}(\hat{\mathbf{x}}-\hat{\mathbf{y}}+\hat{\mathbf{z}}) ; \hat{\mathbf{k}}\right) \\
& \left.-\mathcal{H}_{3}^{a n t}\left(\frac{1}{\sqrt{3}}(\hat{\mathbf{x}}-\hat{\mathbf{y}}-\hat{\mathbf{z}}) ; \hat{\mathbf{k}}\right)\right\} .
\end{aligned}
$$

It is interesting to notice that we have determined seven independent generalized moments of the type $\widetilde{\mathcal{M}}_{0}^{(3, j)}(j=0,1, \ldots, 7)$, and every other moment of the same type corresponding to another cartesian harmonic of third degree is linearly dependent on them and so easily reducible from them as a linear combination.

It is apparent that when the degree of the harmonic functions entering the integrand in the definition of the moments increases, the number of the necessary observation points augments. An interesting question arises: Is it necessary to restrict ourselves to generalized moments referring only to the fundamental component $\Phi_{0}$ of the lowfrequency approximation (7) of the total field? If the answer is negative, a second question emerges: Is there any profit produced by exploiting higher-order components of the expansion (7)? The answers to these simple questions is of fundamental importance for the solution of the inverse scattering problem. As a matter of fact, this work and the forthcoming inversion algorithm [6] aim at giving strong evidence that it is indeed possible to exploit the data pertaining to higher-order components in the low-frequency approximation and so establish a qualitative broadening as well as a strong diversification with the primitive method proposed in [3]. It seems that the exploitation of the moments concerning the components $\Phi_{i}(\mathbf{r} ; \hat{\mathbf{k}})$ (with $i>0$ ) contributes, at least, to diminish the set of the needed observation points accordingly. In addition, it is already known that in contrast to the zeroth-order approximation $\Phi_{0}(\mathbf{r})$, the higher-order terms involve the excitation orientation $\hat{\mathbf{k}}$, so giving a rich parametric improvement to the characteristics of the measurement data. We are now in position to stimulate the scatterer from several directions, and this geometrical feature is of course inscribed in the far-field pattern, rendering the data more "informative" for the sought reconstruction. The moments and the underlying fields of higher order merit special treatment. 
5. Fields and moments of higher order. In the framework of exploring the regime of $\Phi_{i}(\mathbf{r} ; \hat{\mathbf{k}}), i \geq 1$, we start with the next proposition characterizing the first-order component:

Proposition 7. It follows that $\Phi_{1}(\mathbf{r} ; \hat{\mathbf{k}})=-M_{0}^{0} \Phi_{0}(\mathbf{r})+\hat{\mathbf{k}} \cdot \mathbf{A}(\mathbf{r})$, where $\mathbf{A}(\mathbf{r})$ satisfies the integral representation

$$
\mathbf{A}(\mathbf{r})=\mathbf{r}-\frac{1}{4 \pi} \int_{\partial D} \frac{1}{\left|\mathbf{r}-\mathbf{r}^{\prime}\right|} \frac{\partial \mathbf{A}}{\partial n^{\prime}}\left(\mathbf{r}^{\prime}\right) d s\left(\mathbf{r}^{\prime}\right), \quad \mathbf{r} \in R^{3} \backslash D .
$$

Furthermore

$$
\int_{\partial D} \frac{\partial \mathbf{A}}{\partial n}(\mathbf{r}) d s(\mathbf{r})=\int_{\partial D} \mathbf{r} \frac{\partial \Phi_{0}}{\partial n}(\mathbf{r}) d s(\mathbf{r}) .
$$

Proof. The first-order component $\Phi_{1}(\mathbf{r} ; \hat{\mathbf{k}})$ can be expressed in terms of spherical harmonics with respect to the excitation orientation argument $\hat{\mathbf{k}}$ :

$$
\Phi_{1}(\mathbf{r} ; \hat{\mathbf{k}})=\Phi_{1}^{(0)}(\mathbf{r})+\hat{\mathbf{k}} \cdot \mathbf{A}(\mathbf{r})+\sum_{n=2}^{\infty} \sum_{m=-n}^{n} \frac{(n-|m|) !}{(n+|m|) !} \overline{Y_{n}^{m}(\hat{\mathbf{k}})} A_{1}^{(n, m)}(\mathbf{r}) .
$$

The integral representation (8) yields the following form for $n=1$ :

$$
\begin{aligned}
& \Phi_{1}(\mathbf{r} ; \hat{\mathbf{k}})=(\hat{\mathbf{k}} \cdot \mathbf{r})-\frac{1}{4 \pi} \int_{\partial D} \frac{1}{\left|\mathbf{r}-\mathbf{r}^{\prime}\right|} \frac{\partial \Phi_{1}}{\partial n^{\prime}}\left(\mathbf{r}^{\prime} ; \hat{\mathbf{k}}\right) d s\left(\mathbf{r}^{\prime}\right) \\
& -\frac{1}{4 \pi} \int_{\partial D} \frac{\partial \Phi_{0}}{\partial n^{\prime}}\left(\mathbf{r}^{\prime}\right) d s\left(\mathbf{r}^{\prime}\right), \quad \mathbf{r} \in R^{3} \backslash D .
\end{aligned}
$$

Inserting expansion (49) into the representation (50) and using orthogonality of the spherical harmonics we obtain the following series of integral equations:

$$
\begin{aligned}
\Phi_{1}^{(0)}(\mathbf{r})= & -M_{0}^{0}-\frac{1}{4 \pi} \int_{\partial D} \frac{1}{\left|\mathbf{r}-\mathbf{r}^{\prime}\right|} \frac{\partial \Phi_{1}^{(0)}}{\partial n^{\prime}}\left(\mathbf{r}^{\prime}\right) d s\left(\mathbf{r}^{\prime}\right), \\
\mathbf{A}(\mathbf{r})= & \mathbf{r}-\frac{1}{4 \pi} \int_{\partial D} \frac{1}{\left|\mathbf{r}-\mathbf{r}^{\prime}\right|} \frac{\partial \mathbf{A}}{\partial n^{\prime}}\left(\mathbf{r}^{\prime}\right) d s\left(\mathbf{r}^{\prime}\right), \\
A_{1}^{(n, m)}(\mathbf{r})= & -\frac{1}{4 \pi} \int_{\partial D} \frac{1}{\left|\mathbf{r}-\mathbf{r}^{\prime}\right|} \frac{\partial A_{1}^{(n, m)}}{\partial n^{\prime}}\left(\mathbf{r}^{\prime}\right) d s\left(\mathbf{r}^{\prime}\right), n>1,|m| \leq n \\
& \left(\text { for all } \mathbf{r} \in R^{3} \backslash D\right) .
\end{aligned}
$$

In particular,

$$
\Phi_{1}^{(0)}(\mathbf{r})=\mathbf{A}(\mathbf{r})=A_{1}^{(n, m)}(\mathbf{r})=0, \quad \mathbf{r} \in \partial D .
$$

We recall that the Rayleigh approximation $\Phi_{0}$ obeys

$$
\Phi_{0}(\mathbf{r})=1-\frac{1}{4 \pi} \int_{\partial D} \frac{1}{\left|\mathbf{r}-\mathbf{r}^{\prime}\right|} \frac{\partial \Phi_{0}}{\partial n^{\prime}}\left(\mathbf{r}^{\prime}\right) d s\left(\mathbf{r}^{\prime}\right), \quad \mathbf{r} \in R^{3} \backslash D
$$

and particularly $\Phi_{0}(\mathbf{r})=0$ for $\mathbf{r} \in \partial D$. Comparing (52155) and evoking the uniqueness of the exterior Dirichlet Laplace boundary value problem, we infer that

$$
\Phi_{1}^{(0)}(\mathbf{r})=-M_{0}^{0} \Phi_{0}(\mathbf{r}), \quad \mathbf{r} \in R^{3} \backslash D .
$$

The last equation can be derived through an alternative approach, based on the integral equation framework, which is necessary several times in the sequel. More precisely we consider (55) and take the normal derivative on both sides by approaching from outside 
a point $\mathbf{r} \in \partial D$. The well-known relations concerning jump properties of layer potentials [16] are evoked to provide

$$
\frac{1}{2} \frac{\partial \Phi_{0}}{\partial n}(\mathbf{r})=-\frac{1}{4 \pi} \int_{\partial D} \frac{\partial}{\partial n}\left(\frac{1}{\left|\mathbf{r}-\mathbf{r}^{\prime}\right|}\right) \frac{\partial \Phi_{0}}{\partial n^{\prime}}\left(\mathbf{r}^{\prime}\right) d s\left(\mathbf{r}^{\prime}\right), \quad \mathbf{r} \in \partial D .
$$

In terms of the well-known integral operator $\mathbf{K}^{\prime}$ [16] defined by $\left(\mathbf{K}^{\prime} g\right)(\mathbf{r})=2 \frac{1}{4 \pi} \int_{\partial D} \frac{\partial}{\partial n}\left(\frac{1}{\left|\mathbf{r}-\mathbf{r}^{\prime}\right|}\right) g\left(\mathbf{r}^{\prime}\right) d s\left(\mathbf{r}^{\prime}\right)$, (57) is equivalent to stating that the normal derivative $\frac{\partial \Phi_{0}}{\partial n}$ belongs to the nullspace of the operator $I+\mathbf{K}^{\prime}$, i.e.

$$
\left(I+\mathbf{K}^{\prime}\right)\left(\frac{\partial \Phi_{0}}{\partial n}\right)=0, \quad \mathbf{r} \in \partial D .
$$

Due to the second Fredholm alternative, the kernel of $I+\mathbf{K}^{\prime}$ has equal dimension with the kernel of $I+\mathbf{K}$, where $\mathbf{K}$ stands for the double layer potential defined by $(\mathbf{K} g)(\mathbf{r})$ $=2 \frac{1}{4 \pi} \int_{\partial D} \frac{\partial}{\partial n^{\prime}}\left(\frac{1}{\left|\mathbf{r}-\mathbf{r}^{\prime}\right|}\right) g\left(\mathbf{r}^{\prime}\right) d s\left(\mathbf{r}^{\prime}\right)$. But the kernel of $I+\mathbf{K}$ consists of the traces on the boundary $\partial D$ of the harmonic functions defined on $D$ and satisfying the interior homogeneous Neumann problem (see again [16]). Consequently only the constant functions belong to the kernel of $I+\mathbf{K}$, a fact assuring that $\operatorname{dim}\left(I+\mathbf{K}^{\prime}\right)=1$. Returning to equation (58) (or equivalently to (57)), we infer that there is only one solution $\frac{\partial \Phi_{0}}{\partial n}$ modulo multiplicative constants; i.e., there exists a unique well-defined function $w(\mathbf{r})$ such that $\frac{\partial \Phi_{0}}{\partial n}(\mathbf{r})=c w(\mathbf{r})$. The constant ambiguity disappears when the inserting of $\frac{\partial \Phi_{0}}{\partial n}$ in the representation (55) (for $\mathbf{r} \in \partial D$ ) leads to

$$
c^{-1}=\frac{1}{4 \pi} \int_{\partial D} \frac{1}{\left|\mathbf{r}-\mathbf{r}^{\prime}\right|} w\left(\mathbf{r}^{\prime}\right) d s\left(\mathbf{r}^{\prime}\right), \quad \text { for every } \mathbf{r} \in \partial D .
$$

Applying the analysis above to the component $\Phi_{1}^{(0)}$ appearing in (52), we find that $\Phi_{1}^{(0)}(\mathbf{r})=c^{\prime} w(\mathbf{r})$, where now $c^{\prime}=-M_{0}^{0} c$, and inserting this normal derivative to the integral representation (52), we recover once more the basic relation (56). A third application of the integral equation technique to (54) leads to the result:

$$
A_{1}^{(n, m)}(\mathbf{r})=0, \quad n>1,|m| \leq n, \quad \mathbf{r} \in R^{3} \backslash D .
$$

The last equation reflects of course the physical argument according to which no higherorder spherical $\hat{\mathbf{k}}$-harmonics can emerge, when the stimulus of $\Phi_{1}$ disposes of terms until the first-order spherical harmonics.

Recapitulating, the following representation is valid for the component $\Phi_{1}(\mathbf{r} ; \hat{\mathbf{k}})$ :

$$
\Phi_{1}(\mathbf{r} ; \hat{\mathbf{k}})=-M_{0}^{0} \Phi_{0}(\mathbf{r})+\hat{\mathbf{k}} \cdot \mathbf{A}(\mathbf{r}),
$$

where $\mathbf{A}(\mathbf{r})$ obeys (47).

Finally, to obtain (48), we multiply relation (47)) (considered for $\mathbf{r} \in \partial D$ ), with $\frac{\partial \Phi_{0}}{\partial n}$ and then integrate all over $\partial D$, exploiting simultaneously (55) (again considered on the surface $\partial D)$.

REMARK 8. It is obviously deduced from (61148) that

$$
M_{1}^{0}(\hat{\mathbf{k}})=-\left(M_{0}^{0}\right)^{2}+M_{0}^{1}(\hat{\mathbf{k}}) .
$$

In other words, the knowledge of the zeroth-order moment $M_{0}^{1}(\hat{\mathbf{r}})$ in the forward scattering direction $\hat{\mathbf{r}}=\hat{\mathbf{k}}$ is enough for the determination of the moment $M_{1}^{0}(\hat{\mathbf{k}})$ for every 
possible excitation. This result is important since it reveals for the first but not last time that the moments are not independent.

REMARK 9. It is interesting to notice that $4 M_{1}^{1}(\hat{\mathbf{r}} ; \hat{\mathbf{k}})=\mathcal{H}_{2}(-\hat{\mathbf{r}} ; \hat{\mathbf{k}})-\mathcal{H}_{2}(\hat{\mathbf{r}} ; \hat{\mathbf{k}})$ and so $M_{1}^{1}(\hat{\mathbf{r}} ; \hat{\mathbf{k}})$ can be reconstructed from measurements. But, due to (61), $M_{1}^{1}(\hat{\mathbf{r}} ; \hat{\mathbf{k}})=$ $-M_{0}^{0} M_{0}^{1}(\hat{\mathbf{r}})+\hat{\mathbf{r}} \cdot \widetilde{\mathbf{C}}_{1} \cdot \hat{\mathbf{k}}$, where the tensor $\widetilde{\mathbf{C}}_{1}=\frac{1}{4 \pi} \int_{\partial D} \mathbf{r} \frac{\partial \mathbf{A}}{\partial n}(\mathbf{r}) d s(\mathbf{r})$ is symmetric due to reciprocity.

We keep on by decomposing the second-order component of the total field in terms of harmonics with respect to the excitation orientation $\hat{\mathbf{k}}$ :

Proposition 10. The second-order component $\Phi_{2}(\mathbf{r} ; \hat{\mathbf{k}})$ of the low-frequency approximation of the total field is expanded as follows:

$$
\Phi_{2}(\mathbf{r} ; \hat{\mathbf{k}})=\Phi_{2}^{(0)}(\mathbf{r})+\hat{\mathbf{k}} \cdot \mathbf{A}_{2}(\mathbf{r})+\frac{2}{3} \sum_{m=-2}^{2} \frac{(2-|m|) !}{(2+|m|) !} \overline{Y_{2}^{m}(\hat{\mathbf{k}})} A_{2}^{(2, m)}(\mathbf{r}),
$$

where all the involved components vanish on $\partial D$. Furthermore,

$$
\begin{aligned}
& \frac{1}{4 \pi} \int_{\partial D} \frac{\partial A_{2}^{(2, m)}}{\partial n}(\mathbf{r}) d s(\mathbf{r})=\mathcal{M}_{0}^{(2, m)}, \quad|m| \leq 2, \\
& \mathbf{A}_{2}(\mathbf{r})=-\left\{\frac{1}{2 \pi} \int_{\partial D} \mathbf{r}^{\prime} \frac{\partial \Phi_{0}}{\partial n^{\prime}}\left(\mathbf{r}^{\prime}\right) d s\left(\mathbf{r}^{\prime}\right)\right\} \Phi_{0}(\mathbf{r})
\end{aligned}
$$

while $\Phi_{2}^{(0)}(\mathbf{r})$ satisfies the integral equation

$$
\begin{aligned}
\Phi_{2}^{(0)}(\mathbf{r})= & \frac{r^{2}}{3}-\frac{1}{4 \pi} \int_{\partial D} \frac{1}{\left|\mathbf{r}-\mathbf{r}^{\prime}\right|} \frac{\partial \Phi_{2}^{(0)}}{\partial n^{\prime}}\left(\mathbf{r}^{\prime}\right) d s\left(\mathbf{r}^{\prime}\right)-\frac{1}{2 \pi} \int_{\partial D} \frac{\partial \Phi_{1}^{(0)}}{\partial n^{\prime}}\left(\mathbf{r}^{\prime}\right) d s\left(\mathbf{r}^{\prime}\right) \\
& -\frac{1}{4 \pi} \int_{\partial D}\left|\mathbf{r}-\mathbf{r}^{\prime}\right| \frac{\partial \Phi_{0}}{\partial n^{\prime}}\left(\mathbf{r}^{\prime}\right) d s\left(\mathbf{r}^{\prime}\right) .
\end{aligned}
$$

Proof. The vanishing of all components on $\partial D$ is obvious. Starting from the representation (8) (with $n=2$ ), expanding the excitation term $(\hat{\mathbf{k}} \cdot \mathbf{r})^{2}$ (use (26) for $n=2$ ) together with the fields $\Phi_{2}(\mathbf{r} ; \hat{\mathbf{k}}), \Phi_{1}(\mathbf{r} ; \hat{\mathbf{k}})$ in terms of $\hat{\mathbf{k}}$-harmonics and finally exploiting again the orthogonality of harmonics, we obtain (66) along with

$$
\begin{aligned}
\mathbf{A}_{2}(\mathbf{r}) & =-\frac{1}{4 \pi} \int_{\partial D} \frac{1}{\left|\mathbf{r}-\mathbf{r}^{\prime}\right|} \frac{\partial \mathbf{A}_{2}}{\partial n^{\prime}}\left(\mathbf{r}^{\prime}\right) d s\left(\mathbf{r}^{\prime}\right)-\frac{1}{2 \pi} \int_{\partial D} \frac{\partial \mathbf{A}}{\partial n^{\prime}}\left(\mathbf{r}^{\prime}\right) d s\left(\mathbf{r}^{\prime}\right), \\
A_{2}^{(2, m)}(\mathbf{r}) & =r^{2} Y_{2}^{m}(\hat{\mathbf{r}})-\frac{1}{4 \pi} \int_{\partial D} \frac{1}{\left|\mathbf{r}-\mathbf{r}^{\prime}\right|} \frac{\partial A_{2}^{(2, m)}}{\partial n^{\prime}}\left(\mathbf{r}^{\prime}\right) d s\left(\mathbf{r}^{\prime}\right) \quad|m| \leq 2
\end{aligned}
$$

(for all $\mathbf{r} \in R^{3} \backslash D$ ).

Restricting (68) on $\partial D$, multiplying with $\frac{\partial \Phi_{0}}{\partial n}$ and integrating over $\partial D$, we obtain (64) (by using again representation (55) on the scatterer's surface). In addition, (67) is the vector analogue of (52) and so adopting the argumentation of Proposition 7 and using (48) we form (65).

REMARK 11. It is easy to show, using (64]65) of the last lemma, that

$$
M_{2}^{0}(\hat{\mathbf{k}})=\frac{1}{4 \pi} \int_{\partial D} \frac{\partial \Phi_{2}^{(0)}}{\partial n^{\prime}}\left(\mathbf{r}^{\prime}\right) d s\left(\mathbf{r}^{\prime}\right)-2 M_{0}^{0} M_{0}^{1}(\hat{\mathbf{k}})
$$




$$
+\frac{2}{3} \sum_{m=-2}^{2} \frac{(2-|m|) !}{(2+|m|) !} \overline{Y_{2}^{m}(\hat{\mathbf{k}})} \mathcal{M}_{0}^{(2, m)} .
$$

In addition,

$$
M_{0}^{2}(\hat{\mathbf{r}})=\frac{1}{3} \frac{1}{4 \pi} \int_{\partial D} r^{2} \frac{\partial \Phi_{0}}{\partial n^{\prime}}\left(\mathbf{r}^{\prime}\right) d s\left(\mathbf{r}^{\prime}\right)+\frac{2}{3} \sum_{m=-2}^{2} \frac{(2-|m|) !}{(2+|m|) !} \overline{Y_{2}^{m}(\hat{\mathbf{r}})} \mathcal{M}_{0}^{(2, m)}
$$

So, the measured low-frequency component $\mathcal{H}_{2}^{\text {sym }}$ is written as

$$
\begin{aligned}
\mathcal{H}_{2}^{\text {sym }}(\hat{\mathbf{r}} ; \hat{\mathbf{k}})= & M_{0}^{2}(\hat{\mathbf{r}})+M_{2}^{0}(\hat{\mathbf{k}})=\mathcal{B}+2 \mathcal{H}_{0} \mathcal{H}_{1}^{a n t}(\hat{\mathbf{r}} ; \hat{\mathbf{k}}) \\
& +\frac{2}{3} \sum_{m=-2}^{2} \frac{(2-|m|) !}{(2+|m|) !}\left(\overline{Y_{2}^{m}(\hat{\mathbf{r}})}+\overline{Y_{2}^{m}(\hat{\mathbf{k}})}\right) \mathcal{M}_{0}^{(2, m)},
\end{aligned}
$$

where $4 \pi \mathcal{B}$ is equal to $\int_{\partial D} \frac{\partial \Phi_{2}^{(0)}}{\partial n^{\prime}}\left(\mathbf{r}^{\prime}\right) d s\left(\mathbf{r}^{\prime}\right)+\frac{1}{3} \int_{\partial D}{r^{\prime}}^{2} \frac{\partial \Phi_{0}}{\partial n^{\prime}}\left(\mathbf{r}^{\prime}\right) d s\left(\mathbf{r}^{\prime}\right)$ and recoverable from measurements via (71). However, the quantity $\mathcal{B}$ cannot be separated further. In other words there is not a pure measurement process decomposing $\mathcal{B}$. The generalized moments $\int_{\partial D} \frac{\partial \Phi_{2}^{(0)}}{\partial n} d s$ and $\frac{1}{3} \int_{\partial D} r^{2} \frac{\partial \Phi_{0}}{\partial n} d s$ are condemned to live together participating always in the same summation and the only knowledge about them is exactly their sum! Remember that $\frac{1}{4 \pi} \int_{\partial D} r^{2} \frac{\partial \Phi_{0}}{\partial n} d s$ is exactly $\mathcal{M}_{0}^{(0, m, 2)}$, i.e. belongs to the "resisting" to identification type of moments. There appears for the first time the already mentioned (in the Introduction) bonding between moments, which cannot be broken in applications but, as a concept, lies in the core of the new method presented in this work. Nevertheless, it will be clear in the next section that the members of $\mathcal{B}$ can be estimated via a totally analytic technique, independent of the measurement process.

Passing to moments of third order, the low-frequency approximation $\Phi_{3}$ emerges. A relation of the form (72) is expected to be valid for $\Phi_{3}$, obtained with the same steps followed before:

$$
\begin{aligned}
\Phi_{3}(\mathbf{r})= & \Phi_{3}^{(0)}(\mathbf{r})+\frac{3}{5} \hat{\mathbf{k}} \cdot \mathbf{A}_{3}(\mathbf{r})+\frac{2}{3} \sum_{m=-2}^{2} \frac{(2-|m|) !}{(2+|m|) !} \overline{Y_{2}^{m}(\hat{\mathbf{k}})} A_{3}^{(2, m)}(\mathbf{r}) \\
& +\frac{2}{5} \sum_{m=-3}^{3} \frac{(3-|m|) !}{(3+|m|) !} \overline{Y_{3}^{m}(\hat{\mathbf{k}})} A_{3}^{(3, m)}(\mathbf{r}), \quad \mathbf{r} \in R^{3} \backslash D .
\end{aligned}
$$

Beginning with the representation (8) (with $n=3$ ), exploiting the expansions of the excitation term $(\hat{\mathbf{k}} \cdot \mathbf{r})^{3}$ (use (26) for $n=3$ ) as well as of the fields $\Phi_{1}(\mathbf{r} ; \hat{\mathbf{k}}), \Phi_{2}(\mathbf{r} ; \hat{\mathbf{k}}$ ) and $\Phi_{3}(\mathbf{r} ; \hat{\mathbf{k}})$ in terms of $\hat{\mathbf{k}}$-harmonics and finally using once more the orthogonality of harmonics, we obtain a sequence of relations reminiscent of (66/67/68). We mention, for completeness, the relation involving the vector component $\mathbf{A}_{3}$ :

$$
\begin{aligned}
\mathbf{A}_{3}(\mathbf{r})= & r^{2} \mathbf{r}-\frac{1}{4 \pi} \int_{\partial D} \frac{1}{\left|\mathbf{r}-\mathbf{r}^{\prime}\right|} \frac{\partial \mathbf{A}_{3}}{\partial n^{\prime}}\left(\mathbf{r}^{\prime}\right) d s\left(\mathbf{r}^{\prime}\right)-\frac{5}{4 \pi} \int_{\partial D} \frac{\partial \mathbf{A}_{2}}{\partial n^{\prime}}\left(\mathbf{r}^{\prime}\right) d s\left(\mathbf{r}^{\prime}\right) \\
& -\frac{5}{4 \pi} \int_{\partial D}\left|\mathbf{r}-\mathbf{r}^{\prime}\right| \frac{\partial \mathbf{A}}{\partial n^{\prime}}\left(\mathbf{r}^{\prime}\right) d s\left(\mathbf{r}^{\prime}\right), \mathbf{r} \in R^{3} \backslash D .
\end{aligned}
$$


Treating the integral equation involving $A_{3}^{(3, m)}(\mathbf{r})$, we restrict it on $\partial D$, multiply it with $\frac{\partial \mathbf{A}}{\partial n}$ and integrate it all over the surface to obtain the useful relation

$$
\frac{1}{4 \pi} \int_{\partial D} \mathbf{r} \frac{\partial A_{3}^{(3, m)}}{\partial n}(\mathbf{r}) d s(\mathbf{r})=\frac{1}{4 \pi} \int_{\partial D} r^{3} Y_{3}^{m}(\hat{\mathbf{r}}) \frac{\partial \mathbf{A}}{\partial n}(\mathbf{r}) d s(\mathbf{r}), \quad|m| \leq 3
$$

In addition the relation involving $A_{3}^{(2, m)}$ becomes

$$
A_{3}^{(2, m)}(\mathbf{r})=-\frac{1}{4 \pi} \int_{\partial D} \frac{1}{\left|\mathbf{r}-\mathbf{r}^{\prime}\right|} \frac{\partial A_{3}^{(2, m)}}{\partial n^{\prime}}\left(\mathbf{r}^{\prime}\right) d s\left(\mathbf{r}^{\prime}\right)-\frac{3}{4 \pi} \int_{\partial D} \frac{\partial A_{2}^{(2, m)}}{\partial n^{\prime}}\left(\mathbf{r}^{\prime}\right) d s\left(\mathbf{r}^{\prime}\right),
$$

from which and according to (64), we find that

$$
A_{3}^{(2, m)}(\mathbf{r})=-3 \mathcal{M}_{0}^{(2, m)} \Phi_{0}(\mathbf{r}),|m| \leq 2, \quad \mathbf{r} \in R^{3} \backslash D .
$$

It would be essential to give the next proposition to present how the low-frequency components develop a strong dependence when we pass to higher-order terms. This interrelation is valuable for the evolution of the inversion method.

Proposition 12. (i) The components $\Phi_{0}, \Phi_{2}^{(0)}, \Phi_{3}^{(0)}$ and $\mathbf{A}$ satisfy

$$
\Phi_{3}^{(0)}(\mathbf{r})+3 M_{0}^{0} \Phi_{2}^{(0)}(\mathbf{r})-2 \mathbf{A}(\mathbf{r}) \cdot \mathbf{M}_{0}^{1}=3\left(2\left(M_{0}^{0}\right)^{3}-\mathcal{B}\right) \Phi_{0}(\mathbf{r}), \quad \mathbf{r} \in R^{3} \backslash D .
$$

(ii) The components $\Phi_{2}^{(0)}, \Phi_{4}^{(0)}, \Phi_{5}^{(0)}$ and $\mathbf{A}_{3}$ are interrelated as follows:

$$
\Phi_{5}^{(0)}(\mathbf{r})+5 M_{0}^{0} \Phi_{4}^{(0)}(\mathbf{r})-4 \mathbf{A}_{3}(\mathbf{r}) \cdot \mathbf{M}_{0}^{1}=30\left(2\left(M_{0}^{0}\right)^{3}-\mathcal{B}\right) \Phi_{2}^{(0)}(\mathbf{r}), \quad \mathbf{r} \in R^{3} \backslash D,
$$

where $\mathbf{M}_{0}^{1}$ is the constant vector $\frac{1}{4 \pi} \int_{\partial D} \mathbf{r} \frac{\partial \Phi_{0}}{\partial n}(\mathbf{r}) d s(\mathbf{r})$.

Proof. (i) After some straightforward but extended manipulations, it is proved that the function $\mathcal{G}=\Phi_{3}^{(0)}+3 M_{0}^{0} \Phi_{2}^{(0)}-2 \mathbf{A} \cdot \mathbf{M}_{0}^{1}$ satisfies the integral equation

$$
\mathcal{G}(\mathbf{r})=3\left(2\left(M_{0}^{0}\right)^{3}-\mathcal{B}\right)-\frac{1}{4 \pi} \int_{\partial D} \frac{1}{\left|\mathbf{r}-\mathbf{r}^{\prime}\right|} \frac{\partial \mathcal{G}}{\partial n^{\prime}}\left(\mathbf{r}^{\prime}\right) d s\left(\mathbf{r}^{\prime}\right), \quad \mathbf{r} \in R^{3} \backslash D,
$$

which is sufficient to establish what the lemma states, as explained several times before.

(ii) Exploiting the differential properties of the participant functions, outside $\bar{D}$, we find that

$$
\Delta\left(\Phi_{5}^{(0)}(\mathbf{r})+5 M_{0}^{0} \Phi_{4}^{(0)}(\mathbf{r})-4 \mathbf{A}_{3}(\mathbf{r}) \cdot \mathbf{M}_{0}^{1}\right)=30\left(2\left(M_{0}^{0}\right)^{3}-\mathcal{B}\right)\left(2 \Phi_{0}(\mathbf{r})\right) .
$$

Then the function $\frac{1}{30\left(2\left(M_{0}^{0}\right)^{3}-\mathcal{B}\right)}\left(\Phi_{5}^{(0)}(\mathbf{r})+5 M_{0}^{0} \Phi_{4}^{(0)}(\mathbf{r})-4 \mathbf{A}_{3}(\mathbf{r}) \cdot \mathbf{M}_{0}^{1}\right)$ has the same differential, boundary and asymptotic properties with the function $\Phi_{2}^{(0)}$ and so coincides with it.

The methodology presented in Proposition 12 can of course be generalized to components of higher order.

As mentioned before, in this work we prove that we are in a position to measure moments pertaining to higher-order components of the total field. The first evidence has been given in Lemma 4, where $M_{1}^{0}(\hat{\mathbf{k}})$ has been extracted from measurements. In addition we find simply that $M_{1}^{1}(\hat{\mathbf{r}} ; \hat{\mathbf{k}})=-\frac{1}{2} \mathcal{H}_{2}^{\text {ant }}(\hat{\mathbf{r}} ; \hat{\mathbf{k}})$. The following remark gives more information. 
REMARK 13. The moments of second-order terms, pertaining to the vector component A of $\Phi_{1}$, are calculated in accordance with Lemma [5] More precisely, the starting point is the decomposition $3 M_{1}^{2}(\hat{\mathbf{r}} ; \hat{\mathbf{k}})+M_{3}^{0}(\hat{\mathbf{r}} ; \hat{\mathbf{k}})=-\mathcal{H}_{3}^{\text {sym }}(\hat{\mathbf{r}} ; \hat{\mathbf{k}})$. We eliminate $M_{3}^{0}$ by subtracting measurements in rectangular observations $\hat{\mathbf{r}}_{i}$, obtaining

$$
3 M_{1}^{2}\left(\hat{\mathbf{r}}_{i} ; \hat{\mathbf{k}}\right)-3 M_{1}^{2}\left(\hat{\mathbf{r}}_{j} ; \hat{\mathbf{k}}\right)=-\mathcal{H}_{3}^{\text {sym }}\left(\hat{\mathbf{r}}_{i} ; \hat{\mathbf{k}}\right)+\mathcal{H}_{3}^{\text {sym }}\left(\hat{\mathbf{r}}_{j} ; \hat{\mathbf{k}}\right) .
$$

Using the representation $\Phi_{1}(\mathbf{r} ; \hat{\mathbf{k}})=-M_{0}^{0} \Phi_{0}(\mathbf{r})+\hat{\mathbf{k}} \cdot \mathbf{A}(\mathbf{r})$, we can find all the moments of the type $\int_{\partial D} h_{2, j}\left(\mathbf{r}^{\prime}\right) \frac{\partial \mathbf{A}}{\partial n^{\prime}}\left(\mathbf{r}^{\prime}\right) d s\left(\mathbf{r}^{\prime}\right)$. As an example,

$$
\begin{aligned}
& 3 \frac{1}{4 \pi} \int_{\partial D}\left(x^{2}-y^{2}\right) \frac{\partial \mathbf{A}}{\partial n}(\mathbf{r}) d s(\mathbf{r}) \cdot \hat{\mathbf{k}}=-\mathcal{H}_{3}^{s y m}(\hat{\mathbf{x}} ; \hat{\mathbf{k}})+\mathcal{H}_{3}^{s y m}(\hat{\mathbf{y}} ; \hat{\mathbf{k}}) \\
& +3 M_{0}^{0} \frac{1}{4 \pi} \int_{\partial D}\left(x^{2}-y^{2}\right) \frac{\partial \Phi_{0}}{\partial n}(\mathbf{r}) d s(\mathbf{r})
\end{aligned}
$$

where the last term (referring to the component $\Phi_{0}$ ) belongs to the type of moments determined in Lemma 5 ,

The case of moments of third order referring to $\mathbf{A}$ is complex and merits to be presented:

Proposition 14. The moments of third order generated by $\mathbf{A}$ are determined from measurements.

Proof. We start by considering $\mathcal{H}_{4}(\hat{\mathbf{r}} ; \hat{\mathbf{k}})$ :

$$
\mathcal{H}_{4}(\hat{\mathbf{r}} ; \hat{\mathbf{k}})=M_{4}^{0}(\hat{\mathbf{k}})-4 M_{3}^{1}(\hat{\mathbf{r}} ; \hat{\mathbf{k}})+6 M_{2}^{2}(\hat{\mathbf{r}} ; \hat{\mathbf{k}})-4 M_{1}^{3}(\hat{\mathbf{r}} ; \hat{\mathbf{k}})+M_{0}^{4}(\hat{\mathbf{r}}) .
$$

We deduce that

$$
M_{3}^{1}(\hat{\mathbf{r}} ; \hat{\mathbf{k}})+M_{1}^{3}(\hat{\mathbf{r}} ; \hat{\mathbf{k}})=-\frac{1}{4} \mathcal{H}_{4}^{a n t}(\hat{\mathbf{r}} ; \hat{\mathbf{k}}),
$$

where the antisymmetric part $\mathcal{H}_{4}^{a n t}$ is defined similarly as several times before. Inserting the $\hat{\mathbf{k}}$-expansion of the underlying fields in (84), considering a new equation produced by the opposite excitation $(-\hat{\mathbf{k}})$ and subtracting the two equations, we obtain

$$
\begin{aligned}
& \frac{1}{4 \pi}\left\{\frac{3}{10} \int_{\partial D}\left(\hat{\mathbf{r}} \cdot \mathbf{r}^{\prime}\right) \frac{\partial \mathbf{A}_{3}}{\partial n^{\prime}}\left(\mathbf{r}^{\prime}\right) d s\left(\mathbf{r}^{\prime}\right) \cdot \hat{\mathbf{k}}+\frac{1}{5} \sum_{m=-3}^{3} \frac{(3-|m|) !}{(3+|m|) !} Y_{3}^{m}(\hat{\mathbf{k}})\right. \\
& \left.\times\left(\int_{\partial D}\left(\hat{\mathbf{r}} \cdot \mathbf{r}^{\prime}\right) \frac{\partial A_{3}^{(3, m)}}{\partial n^{\prime}}\left(\mathbf{r}^{\prime}\right) d s\left(\mathbf{r}^{\prime}\right)\right)+\frac{1}{2} \int_{\partial D}\left(\hat{\mathbf{r}} \cdot \mathbf{r}^{\prime}\right)^{3} \frac{\partial \mathbf{A}}{\partial n^{\prime}}\left(\mathbf{r}^{\prime}\right) d s\left(\mathbf{r}^{\prime}\right) \cdot \hat{\mathbf{k}}\right\} \\
& =-\frac{1}{16}\left(\mathcal{H}_{4}^{a n t}(\hat{\mathbf{r}} ; \hat{\mathbf{k}})-\mathcal{H}_{4}^{a n t}(\hat{\mathbf{r}} ;-\hat{\mathbf{k}})\right) .
\end{aligned}
$$

Expanding $\left(\hat{\mathbf{r}} \cdot \mathbf{r}^{\prime}\right)^{3}$ in terms of spherical harmonics and using (74) we transform (85) in the form

$$
\begin{aligned}
& \frac{3}{10} \hat{\mathbf{r}} \cdot\left\{\int_{\partial D}\left(\mathbf{r}^{\prime} \frac{\partial \mathbf{A}_{3}}{\partial n^{\prime}}\left(\mathbf{r}^{\prime}\right)+{r^{\prime}}^{2} \mathbf{r}^{\prime} \frac{\partial \mathbf{A}}{\partial n^{\prime}}\left(\mathbf{r}^{\prime}\right)\right) d s\left(\mathbf{r}^{\prime}\right)\right\} \cdot \hat{\mathbf{k}} \\
& +\frac{1}{5} \sum_{m=-3}^{3} \frac{(3-|m|) !}{(3+|m|) !}\left(\overline{Y_{3}^{m}(\hat{\mathbf{k}}) \hat{\mathbf{r}}}+\overline{Y_{3}^{m}(\hat{\mathbf{r}})} \hat{\mathbf{k}}\right) \cdot \int_{\partial D} r^{\prime 3} Y_{3}^{m}\left(\hat{\mathbf{r}}^{\prime}\right) \frac{\partial \mathbf{A}}{\partial n^{\prime}}\left(\mathbf{r}^{\prime}\right) d s\left(\mathbf{r}^{\prime}\right) \\
& =-\frac{1}{4} \pi\left(\mathcal{H}_{4}^{a n t}(\hat{\mathbf{r}} ; \hat{\mathbf{k}})-\mathcal{H}_{4}^{a n t}(\hat{\mathbf{r}} ;-\hat{\mathbf{k}})\right) .
\end{aligned}
$$


We denote generally

$$
\hat{\mathcal{H}}_{n}^{a n t}(\hat{\mathbf{r}} ; \hat{\mathbf{k}})=\frac{1}{2}\left(\mathcal{H}_{n}^{a n t}(\hat{\mathbf{r}} ; \hat{\mathbf{k}})-\mathcal{H}_{n}^{a n t}(\hat{\mathbf{r}} ;-\hat{\mathbf{k}})\right) .
$$

We apply (86) for three orientations forming an orthonormal basis $\hat{\mathbf{r}}_{i}, i=1,2,3 \in R^{3}$ and for three perpendicular excitations building the basis $\hat{\mathbf{k}}_{j}, j=1,2,3 \in R^{3}$. Multiplying each relation with the corresponding dyadic $\hat{\mathbf{r}}_{i} \hat{\mathbf{k}}_{j}$, while considering the same sets $\hat{\mathbf{r}}_{i}=$ $\hat{\mathbf{k}}_{i}, i=1,2,3$ and finally adding over $i, j=1,2,3$, we obtain

$$
\begin{aligned}
& \frac{3}{10}\left\{\int_{\partial D}\left(\mathbf{r}^{\prime} \frac{\partial \mathbf{A}_{3}}{\partial n^{\prime}}\left(\mathbf{r}^{\prime}\right)+r^{\prime}{ }^{\prime} \mathbf{r}^{\prime} \frac{\partial \mathbf{A}}{\partial n^{\prime}}\left(\mathbf{r}^{\prime}\right)\right) d s\left(\mathbf{r}^{\prime}\right)\right\} \\
& +\frac{2}{5} \sum_{m=-3}^{3} \frac{(3-|m|) !}{(3+|m|) !} \sum_{i=1}^{3}\left(\hat{\mathbf{r}}_{i} \overline{Y_{3}^{m}\left(\hat{\mathbf{r}}_{i}\right)}\right) \int_{\partial D}{r^{\prime}}^{3} Y_{3}^{m}\left(\hat{\mathbf{r}}^{\prime}\right) \frac{\partial \mathbf{A}}{\partial n^{\prime}}\left(\mathbf{r}^{\prime}\right) d s\left(\mathbf{r}^{\prime}\right) \\
& =-\frac{\pi}{2} \sum_{i=1}^{3} \sum_{j=1}^{3}\left[\hat{\mathbf{r}}_{i} \hat{\mathcal{H}}_{4}^{a n t}\left(\hat{\mathbf{r}}_{i} ; \hat{\mathbf{r}}_{j}\right) \hat{\mathbf{r}}_{j}\right] .
\end{aligned}
$$

Following the same manipulations as in Proposition 6 (the technique exactly after (35)), it is proved that (88) can provide with all the necessary moments of the type $\int_{\partial D} r^{\prime 3} Y_{3}^{m}\left(\hat{\mathbf{r}}^{\prime}\right) \frac{\partial \mathbf{A}}{\partial n^{\prime}}\left(\mathbf{r}^{\prime}\right) d s\left(\mathbf{r}^{\prime}\right)$ or the equivalent set of moments of the type $\int_{\partial D} h_{3, j}\left(\mathbf{r}^{\prime}\right) \frac{\partial A_{l}}{\partial n^{\prime}}\left(\mathbf{r}^{\prime}\right) d s\left(\mathbf{r}^{\prime}\right)$, where $A_{l}, l=1,2,3$ are the components of the vector $\mathbf{A}$ and $h_{3, j}\left(\mathbf{r}^{\prime}\right)(j=1,2, \ldots, 7)$ stand for an independent set of cartesian harmonic functions (the same met in Proposition 6). So, in accordance with (40[41), we find that

$$
\begin{aligned}
& \frac{1}{4 \pi} \int_{\partial D}\left(x^{3}-3 x y^{2}\right) \frac{\partial \mathbf{A}}{\partial n}(\mathbf{r}) d s(\mathbf{r})=\frac{1}{8}\left\{\hat{\mathcal{H}}_{4}^{a n t}\left(\frac{1}{\sqrt{2}}(\hat{\mathbf{x}}+\hat{\mathbf{y}}) ; \frac{1}{\sqrt{2}}(\hat{\mathbf{x}}+\hat{\mathbf{y}})\right)(\hat{\mathbf{x}}+\hat{\mathbf{y}})\right. \\
&+\hat{\mathcal{H}}_{4}^{a n t}\left(\frac{1}{\sqrt{2}}(\hat{\mathbf{x}}+\hat{\mathbf{y}}) ; \frac{1}{\sqrt{2}}(\hat{\mathbf{x}}-\hat{\mathbf{y}})\right)(\hat{\mathbf{x}}-\hat{\mathbf{y}})+\sqrt{2} \hat{\mathcal{H}}_{4}^{a n t}\left(\frac{1}{\sqrt{2}}(\hat{\mathbf{x}}+\hat{\mathbf{y}}) ; \hat{\mathbf{z}}\right) \hat{\mathbf{z}} \\
&+\hat{\mathcal{H}}_{4}^{a n t}\left(\frac{1}{\sqrt{2}}(\hat{\mathbf{x}}-\hat{\mathbf{y}}) ; \frac{1}{\sqrt{2}}(\hat{\mathbf{x}}+\hat{\mathbf{y}})\right)(\hat{\mathbf{x}}+\hat{\mathbf{y}})+\sqrt{2} \hat{\mathcal{H}}_{4}^{a n t}\left(\frac{1}{\sqrt{2}}(\hat{\mathbf{x}}-\hat{\mathbf{y}}) ; \hat{\mathbf{z}}\right) \hat{\mathbf{z}} \\
&+\hat{\mathcal{H}}_{4}^{a n t}\left(\frac{1}{\sqrt{2}}(\hat{\mathbf{x}}-\hat{\mathbf{y}}) ; \frac{1}{\sqrt{2}}(\hat{\mathbf{x}}-\hat{\mathbf{y}})\right)(\hat{\mathbf{x}}-\hat{\mathbf{y}})-2 \hat{\mathcal{H}}_{4}^{a n t}(\hat{\mathbf{x}} ; \hat{\mathbf{x}}) \hat{\mathbf{x}} \\
&\left.-2 \hat{\mathcal{H}}_{4}^{a n t}(\hat{\mathbf{x}} ; \hat{\mathbf{y}}) \hat{\mathbf{y}}-2 \hat{\mathcal{H}}_{4}^{a n t}(\hat{\mathbf{x}} ; \hat{\mathbf{z}}) \hat{\mathbf{z}}\right\}, \\
& \frac{1}{4 \pi} \int_{\partial D}\left(y^{3}-3 y x^{2}\right) \frac{\partial \mathbf{A}}{\partial n}(\mathbf{r}) d s(\mathbf{r})=\frac{1}{8}\left\{\hat{\mathcal{H}}_{4}^{a n t}\left(\frac{1}{\sqrt{2}}(\hat{\mathbf{x}}+\hat{\mathbf{y}}) ; \frac{1}{\sqrt{2}}(\hat{\mathbf{x}}+\hat{\mathbf{y}})\right)(\hat{\mathbf{x}}+\hat{\mathbf{y}})\right. \\
& \quad+\hat{\mathcal{H}}_{4}^{a n t}\left(\frac{1}{\sqrt{2}}(\hat{\mathbf{x}}+\hat{\mathbf{y}}) ; \frac{1}{\sqrt{2}}(\hat{\mathbf{x}}-\hat{\mathbf{y}})\right)(\hat{\mathbf{x}}-\hat{\mathbf{y}})+\sqrt{2} \hat{\mathcal{H}}_{4}^{a n t}\left(\frac{1}{\sqrt{2}}(\hat{\mathbf{x}}+\hat{\mathbf{y}}) ; \hat{\mathbf{z}}\right) \hat{\mathbf{z}} \\
& \quad-\hat{\mathcal{H}}_{4}^{a n t}\left(\frac{1}{\sqrt{2}}(\hat{\mathbf{x}}-\hat{\mathbf{y}}) ; \frac{1}{\sqrt{2}}(\hat{\mathbf{x}}+\hat{\mathbf{y}})\right)(\hat{\mathbf{x}}+\hat{\mathbf{y}})-\sqrt{2} \hat{\mathcal{H}}_{4}^{a n t}\left(\frac{1}{\sqrt{2}}(\hat{\mathbf{x}}-\hat{\mathbf{y}}) ; \hat{\mathbf{z}}\right) \hat{\mathbf{z}} \\
& \quad-\hat{\mathcal{H}}_{4}^{a n t}\left(\frac{1}{\sqrt{2}}(\hat{\mathbf{x}}-\hat{\mathbf{y}}) ; \frac{1}{\sqrt{2}}(\hat{\mathbf{x}}-\hat{\mathbf{y}})\right)(\hat{\mathbf{x}}-\hat{\mathbf{y}})-2 \hat{\mathcal{H}}_{4}^{a n t}(\hat{\mathbf{y}} ; \hat{\mathbf{x}}) \hat{\mathbf{x}} \\
&\left.\quad-2 \hat{\mathcal{H}}_{4}^{a n t}(\hat{\mathbf{y}} ; \hat{\mathbf{y}}) \hat{\mathbf{y}}-2 \hat{\mathcal{H}}_{4}^{a n t}(\hat{\mathbf{y}} ; \hat{\mathbf{z}}) \hat{\mathbf{z}}\right\} .
\end{aligned}
$$


Reciprocity relations for the low-frequency components $\hat{\mathcal{H}}_{4}^{a n t}$ can be used to prove the mirror $(\hat{\mathbf{x}} \leftrightarrow \hat{\mathbf{y}})$ relationship between the representations above. Following cyclic permutations of $(\hat{\mathbf{x}}, \hat{\mathbf{y}}, \hat{\mathbf{z}})$, exactly as in Lemma 4, we can also define the moments $\frac{1}{4 \pi} \int_{\partial D}\left(y^{3}-\right.$ $\left.3 y z^{2}\right) \frac{\partial \mathbf{A}}{\partial n}(\mathbf{r}) d s(\mathbf{r}), \frac{1}{4 \pi} \int_{\partial D}\left(z^{3}-3 z y^{2}\right) \frac{\partial \mathbf{A}}{\partial n}(\mathbf{r}) d s(\mathbf{r}), \quad \frac{1}{4 \pi} \int_{\partial D}\left(z^{3}-3 z x^{2}\right) \frac{\partial \mathbf{A}}{\partial n}(\mathbf{r}) d s(\mathbf{r}) \quad$ and $\frac{1}{4 \pi} \int_{\partial D}\left(x^{3}-3 x z^{2}\right) \frac{\partial \mathbf{A}}{\partial n}(\mathbf{r}) d s(\mathbf{r})$. The last moment $\frac{1}{4 \pi} \int_{\partial D} x y z \frac{\partial \mathbf{A}}{\partial n}(\mathbf{r}) d s(\mathbf{r})$ can be defined as in Proposition 6, but no use of it will be needed.

The reciprocity property $\mathcal{H}_{n}(\hat{\mathbf{r}} ; \hat{\mathbf{k}})=\mathcal{H}_{n}(-\hat{\mathbf{k}} ;-\hat{\mathbf{r}})$ has been just mentioned before and presents a lot of very interesting consequences. Actually, what has been evoked in the previous lemma is the reciprocity entailed (as can easily be shown) from the original measurements to the functions $\hat{\mathcal{H}}_{n}^{\text {ant }}$ met frequently in this work.

It is useful to reconstruct more intrinsic moments from measurements, as the following proposition reveals.

Proposition 15. The generalized moments $\int_{\partial D} h_{2, j}(\mathbf{r}) \frac{\partial A_{2}^{(2, m)}}{\partial n}(\mathbf{r}),|m| \leq 2, j=1,2, \ldots, 5$ are recoverable from measurements.

Proof. We refer to the low-frequency component $\mathcal{H}_{4}(\hat{\mathbf{r}} ; \hat{\mathbf{k}})$ of the far-field pattern and obtain easily that

$$
\mathcal{H}_{4}^{\text {sym }}(\hat{\mathbf{r}} ; \hat{\mathbf{k}})=M_{4}^{0}(\hat{\mathbf{k}})+6 M_{2}^{2}(\hat{\mathbf{r}} ; \hat{\mathbf{k}})+M_{0}^{4}(\hat{\mathbf{r}})
$$

or

$$
\begin{aligned}
& \mathcal{H}_{4}^{s y m}(\hat{\mathbf{r}} ; \hat{\mathbf{k}})=\int_{\partial D} \frac{\partial \Phi_{4}}{\partial n^{\prime}}\left(\mathbf{r}^{\prime} ; \hat{\mathbf{k}}\right) d s\left(\mathbf{r}^{\prime}\right)+6 \int_{\partial D}\left(\hat{\mathbf{r}} \cdot \mathbf{r}^{\prime}\right)^{2} \frac{\partial \Phi_{2}^{(0)}}{\partial n^{\prime}}\left(\mathbf{r}^{\prime}\right) d s\left(\mathbf{r}^{\prime}\right) \\
& +4 \sum_{m=-2}^{2} \frac{(2-|m|) !}{(2+|m|) !} \frac{Y_{2}^{m}(\hat{\mathbf{k}})}{(2+\hat{r}} \int_{\partial D}\left(\hat{\mathbf{r}} \cdot \mathbf{r}^{\prime}\right)^{2} \frac{\partial A_{2}^{(2, m)}}{\partial n^{\prime}}\left(\mathbf{r}^{\prime}\right) d s\left(\mathbf{r}^{\prime}\right) \\
& +6 \int_{\partial D}\left(\hat{\mathbf{r}} \cdot \mathbf{r}^{\prime}\right)^{2} \frac{\partial \mathbf{A}_{2}}{\partial n^{\prime}}\left(\mathbf{r}^{\prime}\right) d s\left(\mathbf{r}^{\prime}\right) \cdot \hat{\mathbf{k}}+\int_{\partial D}\left(\hat{\mathbf{r}} \cdot \mathbf{r}^{\prime}\right)^{4} \frac{\partial \Phi_{0}}{\partial n^{\prime}}\left(\mathbf{r}^{\prime}\right) d s\left(\mathbf{r}^{\prime}\right)
\end{aligned}
$$

Introducing

$$
\tilde{\mathcal{H}}_{4}^{\text {sym }}(\hat{\mathbf{r}} ; \hat{\mathbf{k}})=\frac{1}{2}\left(\mathcal{H}_{4}^{\text {sym }}(\hat{\mathbf{r}} ; \hat{\mathbf{k}})+\mathcal{H}_{4}^{\text {sym }}(\hat{\mathbf{r}} ;-\hat{\mathbf{k}})\right),
$$

and an orthogonal triple of unit vectors $\hat{\mathbf{r}}_{\alpha}, \alpha=1,2,3$, we find that

$$
\begin{aligned}
& 6 \int_{\partial D}\left(\left(\hat{\mathbf{r}}_{\alpha} \cdot \mathbf{r}^{\prime}\right)^{2}-\left(\hat{\mathbf{r}}_{\beta} \cdot \mathbf{r}^{\prime}\right)^{2}\right) \frac{\partial \Phi_{2}^{(0)}}{\partial n^{\prime}}\left(\mathbf{r}^{\prime}\right) d s\left(\mathbf{r}^{\prime}\right)+4 \sum_{m=-2}^{2} \frac{(2-|m|) !}{(2+|m|) !} \\
& \times \overline{Y_{2}^{m}(\hat{\mathbf{k}})} \int_{\partial D}\left(\left(\hat{\mathbf{r}}_{\alpha} \cdot \mathbf{r}^{\prime}\right)^{2}-\left(\hat{\mathbf{r}}_{\beta} \cdot \mathbf{r}^{\prime}\right)^{2}\right) \frac{\partial A_{2}^{(2, m)}}{\partial n^{\prime}}\left(\mathbf{r}^{\prime}\right) d s\left(\mathbf{r}^{\prime}\right) \\
& +\int_{\partial D}\left(\left(\hat{\mathbf{r}}_{\alpha} \cdot \mathbf{r}^{\prime}\right)^{4}-\left(\hat{\mathbf{r}}_{\beta} \cdot \mathbf{r}^{\prime}\right)^{4}\right) \frac{\partial \Phi_{0}}{\partial n^{\prime}}\left(\mathbf{r}^{\prime}\right) d s\left(\mathbf{r}^{\prime}\right) \\
& =\tilde{\mathcal{H}}_{4}^{s y m}\left(\hat{\mathbf{r}}_{\alpha} ; \hat{\mathbf{k}}\right)-\tilde{\mathcal{H}}_{4}^{s y m}\left(\hat{\mathbf{r}}_{\beta} ; \hat{\mathbf{k}}\right), \quad \alpha, \beta=1,2,3, \ldots
\end{aligned}
$$

Selecting the vectors $\hat{\mathbf{r}}_{\alpha}$ to coincide with the cartesian unit vectors and to stimulate $\hat{\mathbf{k}}$ in appropriate directions, we may easily recover all moments of type $\int_{\partial D} h_{2, j}(\mathbf{r}) \frac{\partial A_{2}^{(2, m)}}{\partial n}(\mathbf{r})$. 
More precisely, we obtain

$$
\begin{aligned}
& 2 \int_{\partial D}\left(x^{\prime 2}-y^{\prime 2}\right) \Re\left(\frac{\partial A_{2}^{(2,2)}}{\partial n^{\prime}}\left(\mathbf{r}^{\prime}\right)\right) d s\left(\mathbf{r}^{\prime}\right) \\
& =\left(\tilde{\mathcal{H}}_{4}^{s y m}(\hat{\mathbf{x}} ; \hat{\mathbf{x}})-\tilde{\mathcal{H}}_{4}^{s y m}(\hat{\mathbf{y}} ; \hat{\mathbf{x}})\right)-\left(\tilde{\mathcal{H}}_{4}^{\text {sym }}(\hat{\mathbf{x}} ; \hat{\mathbf{y}})-\tilde{\mathcal{H}}_{4}^{\text {sym }}(\hat{\mathbf{y}} ; \hat{\mathbf{y}})\right), \\
& 2 \int_{\partial D}\left(x^{\prime 2}-y^{\prime 2}\right) \Im\left(\frac{\partial A_{2}^{(2,2)}}{\partial n^{\prime}}\left(\mathbf{r}^{\prime}\right)\right) d s\left(\mathbf{r}^{\prime}\right) \\
& =\left(\tilde{\mathcal{H}}_{4}^{s y m}\left(\hat{\mathbf{x}} ; \frac{1}{\sqrt{2}}(\hat{\mathbf{x}}+\hat{\mathbf{y}})\right)-\tilde{\mathcal{H}}_{4}^{\text {sym }}\left(\hat{\mathbf{y}} ; \frac{1}{\sqrt{2}}(\hat{\mathbf{x}}+\hat{\mathbf{y}})\right)\right) \\
& -\left(\tilde{\mathcal{H}}_{4}^{\text {sym }}\left(\hat{\mathbf{x}} ; \frac{1}{\sqrt{2}}(\hat{\mathbf{x}}-\hat{\mathbf{y}})\right)-\tilde{\mathcal{H}}_{4}^{\text {sym }}\left(\hat{\mathbf{y}} ; \frac{1}{\sqrt{2}}(\hat{\mathbf{x}}-\hat{\mathbf{y}})\right)\right), \\
& 4 \int_{\partial D}\left(x^{\prime 2}-y^{\prime 2}\right) \frac{\partial A_{2}^{(2,0)}}{\partial n^{\prime}}\left(\mathbf{r}^{\prime}\right) d s\left(\mathbf{r}^{\prime}\right)=\int_{\partial D}\left(x^{\prime 2}-y^{\prime 2}\right) \Re\left(\frac{\partial A_{2}^{(2,2)}}{\partial n^{\prime}}\left(\mathbf{r}^{\prime}\right)\right) d s\left(\mathbf{r}^{\prime}\right) \\
& +\left(\tilde{\mathcal{H}}_{4}^{\text {sym }}(\hat{\mathbf{x}} ; \hat{\mathbf{z}})-\tilde{\mathcal{H}}_{4}^{\text {sym }}(\hat{\mathbf{y}} ; \hat{\mathbf{z}})\right)-\left(\tilde{\mathcal{H}}_{4}^{\text {sym }}(\hat{\mathbf{x}} ; \hat{\mathbf{x}})-\tilde{\mathcal{H}}_{4}^{\text {sym }}(\hat{\mathbf{y}} ; \hat{\mathbf{x}})\right), \\
& \int_{\partial D}\left(x^{\prime 2}-y^{\prime 2}\right) \Re\left(\frac{\partial A_{2}^{(2,1)}}{\partial n^{\prime}}\left(\mathbf{r}^{\prime}\right)\right) d s\left(\mathbf{r}^{\prime}\right)=\left(\tilde{\mathcal{H}}_{4}^{\text {sym }}\left(\hat{\mathbf{x}} ; \frac{1}{\sqrt{2}}(\hat{\mathbf{x}}+\hat{\mathbf{z}})\right)\right. \\
& \left.-\tilde{\mathcal{H}}_{4}^{\text {sym }}\left(\hat{\mathbf{y}} ; \frac{1}{\sqrt{2}}(\hat{\mathbf{x}}+\hat{\mathbf{z}})\right)\right)-\left(\tilde{\mathcal{H}}_{4}^{\text {sym }}\left(\hat{\mathbf{x}} ; \frac{1}{\sqrt{2}}(\hat{\mathbf{x}}-\hat{\mathbf{z}})\right)\right. \\
& \left.-\tilde{\mathcal{H}}_{4}^{s y m}\left(\hat{\mathbf{y}} ; \frac{1}{\sqrt{2}}(\hat{\mathbf{x}}-\hat{\mathbf{z}})\right)\right) \text {, } \\
& \int_{\partial D}\left(x^{\prime 2}-y^{\prime 2}\right) \Im\left(\frac{\partial A_{2}^{(2,1)}}{\partial n^{\prime}}\left(\mathbf{r}^{\prime}\right)\right) d s\left(\mathbf{r}^{\prime}\right) \\
& =\left(\tilde{\mathcal{H}}_{4}^{\text {sym }}\left(\hat{\mathbf{x}} ; \frac{1}{\sqrt{2}}(\hat{\mathbf{y}}+\hat{\mathbf{z}})\right)-\tilde{\mathcal{H}}_{4}^{\text {sym }}\left(\hat{\mathbf{y}} ; \frac{1}{\sqrt{2}}(\hat{\mathbf{y}}+\hat{\mathbf{z}})\right)\right) \\
& -\left(\tilde{\mathcal{H}}_{4}^{\text {sym }}\left(\hat{\mathbf{x}} ; \frac{1}{\sqrt{2}}(\hat{\mathbf{y}}-\hat{\mathbf{z}})\right)-\tilde{\mathcal{H}}_{4}^{\text {sym }}\left(\hat{\mathbf{y}} ; \frac{1}{\sqrt{2}}(\hat{\mathbf{y}}-\hat{\mathbf{z}})\right)\right) .
\end{aligned}
$$

Cyclic permutation of the observation directions leads to the determination of moments representing "projections" on the harmonics $y^{\prime 2}-z^{\prime 2}$ and $z^{\prime 2}-x^{\prime 2}$. The remaining moments pertaining to the harmonics $x^{\prime} y^{\prime}, y^{\prime} z^{\prime}$ and $z^{\prime} x^{\prime}$ are determined by measuring (and subtracting each time) in the direction pairs $\left(\frac{1}{\sqrt{2}}(\hat{\mathbf{x}}+\hat{\mathbf{y}}), \frac{1}{\sqrt{2}}(\hat{\mathbf{x}}-\hat{\mathbf{y}})\right),\left(\frac{1}{\sqrt{2}}(\hat{\mathbf{y}}+\right.$ $\left.\hat{\mathbf{z}}), \frac{1}{\sqrt{2}}(\hat{\mathbf{y}}-\hat{\mathbf{z}})\right)$ and $\left(\frac{1}{\sqrt{2}}(\hat{\mathbf{z}}+\hat{\mathbf{x}}), \frac{1}{\sqrt{2}}(\hat{\mathbf{z}}-\hat{\mathbf{x}})\right)$ respectively.

A methodology of the same spirit can be applied to $\Phi_{i}$ with $i>3$ and to the underlying moments. However, the corresponding quantitative burden is expected to augment drastically. An essential escort to this tough effort would be the evocation of interrelations of the type introduced in Proposition 12. However, the complete presentation of all this analysis for higher-order terms is out of the scope of this work.

6. Analysis of the second class of moments not directly accessible in measurements. The members of the measured quantity $\mathcal{B}$ are the first resisting to identification moments that already have made their appearance (see Remark 11). The term 
$\mathcal{B}$ that denies to be decomposed, constitutes the mean value of $\mathcal{H}_{2}(\hat{\mathbf{r}}, \hat{\mathbf{k}})$ over $S^{2} \times S^{2}$, while all other participants of $\mathcal{H}_{2}(\hat{\mathbf{r}}, \hat{\mathbf{k}})$ clearly display their building stones.

A fundamental reason for this resisting connection is that the terms participating in $\mathcal{B}$ depend strongly on the position of the coordinate origin, which is not of course a physical but a geometrical feature of the problem.

Lemma 16. Let us stimulate the scatterer by the incident field $\exp (i \mathbf{k} \cdot \mathbf{r}-i \mathbf{k} \cdot \mathbf{d})$ instead of $\exp (i \mathbf{k} \cdot \mathbf{r})$, where $\mathbf{d}$ is some specific translation. The low-frequency series of the new total field obtains the form

$$
u_{n e w}^{\text {tot }}(\mathbf{r} ; \mathbf{k})=\sum_{n=0}^{\infty} \frac{(i k)^{n}}{n !} \widetilde{\Phi}_{n}(\mathbf{r} ; \hat{\mathbf{k}})
$$

where the new components are expressed via the old ones $\left(\Phi_{n}(\mathbf{r} ; \hat{\mathbf{k}})\right)$ as follows:

$$
\widetilde{\Phi}_{n}(\mathbf{r} ; \hat{\mathbf{k}})=\sum_{\rho=0}^{n}(-1)^{\rho}\left(\begin{array}{l}
n \\
\rho
\end{array}\right)(\hat{\mathbf{k}} \cdot \mathbf{d})^{\rho} \Phi_{n-\rho}(\mathbf{r} ; \hat{\mathbf{k}}) .
$$

Proof. Obtaining (98) is a straightforward task.

The important observation is that if we change (translate) variables and consider the new coordinate origin $O^{\prime \prime}$ as the trace of the position vector $\mathbf{d}$, then the new position variable is $\mathbf{r}^{\prime \prime}=\mathbf{r}-\mathbf{d}$ and clearly $u_{\text {new }}^{\text {tot }}\left(\mathbf{r}^{\prime \prime} ; \mathbf{k}\right)=\sum_{n=0}^{\infty} \frac{(i k)^{n}}{n !} \Phi_{n}\left(\mathbf{r}^{\prime \prime} ; \hat{\mathbf{k}}, O^{\prime \prime}\right)$. Then (98) can be used to give the relation

$$
\Phi_{n}\left(\mathbf{r}^{\prime \prime} ; \hat{\mathbf{k}}, O^{\prime \prime}\right)=\sum_{\rho=0}^{n}(-1)^{\rho}\left(\begin{array}{l}
n \\
\rho
\end{array}\right)(\hat{\mathbf{k}} \cdot \mathbf{d})^{\rho} \Phi_{n-\rho}(\mathbf{r} ; \hat{\mathbf{k}}) .
$$

We remark that $\Phi_{0}$ is not affected by the phase shift (or the equivalent coordinate translation) but all the other components are affected. So the initial moment $M_{0}^{1}(\hat{\mathbf{r}})$ is transformed to $M_{0, \text { new }}^{1}(\hat{\mathbf{r}})=M_{0}^{1}(\hat{\mathbf{r}})-(\hat{\mathbf{r}} \cdot \mathbf{d}) M_{0}^{0}$. Selecting

$$
\mathbf{d}=\frac{1}{M_{0}^{0}} \frac{1}{4 \pi} \int_{\partial D} \mathbf{r}^{\prime} \frac{\partial \Phi_{0}}{\partial n^{\prime}}\left(\mathbf{r}^{\prime}\right) d s\left(\mathbf{r}^{\prime}\right)=\frac{1}{M_{0}^{0}} \mathbf{M}_{0}^{1}
$$

we find $M_{0, \text { new }}^{1}(\hat{\mathbf{r}})=0$. So, this particular $\mathbf{d}$ stands for the "physical" center of the scatterer with respect to which the vector moment $M_{0}^{1}$ vanishes, a fact reflecting the annihilation of the weighted mean value of the locations of the scatterer's points $\left(\frac{1}{4 \pi} \int_{\partial D} \mathbf{r}^{\prime \prime} \frac{\partial \Phi_{0}}{\partial n^{\prime \prime}}\left(\mathbf{r}^{\prime \prime}\right) d s\left(\mathbf{r}^{\prime \prime}\right)=0\right)$. We recognize here the well-known result that a shift in the phase of the incident field can be "assigned" to shifting of the coordinate origin. We mention that in the case that the scatterer has inversion symmetry, then the "physical" center $\mathbf{d}$ coincides with the geometrical center of it.

LEMma 17. Translating the coordinate origin from the physical center to a new position, the members $\frac{1}{3} \int_{\partial D} r^{2} \frac{\partial \Phi_{0}}{\partial n}(\mathbf{r}) d s(\mathbf{r}), \int_{\partial D} \frac{\partial \Phi_{2}^{(0)}}{\partial n}(\mathbf{r}) d s(\mathbf{r})$ of $\mathcal{B}$ are augmented equally by the amount $d^{2}(4 \pi) \frac{1}{3} M_{0}^{0}$, where $d$ is the distance between the two coordinate centers. 
Proof. Clearly, the new moment $\frac{1}{3} \int_{\partial D}{r^{\prime}}^{2} \frac{\partial \Phi_{0}}{\partial n^{\prime}}\left(\mathbf{r}^{\prime}, O^{\prime}\right) d s\left(\mathbf{r}^{\prime}\right)$ with respect to the new center $O^{\prime}$ (pointing from the physical center via the location vector $\mathbf{d}=d \hat{\mathbf{d}}$ ) is given by

$$
\begin{aligned}
& \frac{1}{3} \int_{\partial D} r^{\prime 2} \frac{\partial \Phi_{0}}{\partial n^{\prime}}\left(\mathbf{r}^{\prime}, O^{\prime}\right) d s\left(\mathbf{r}^{\prime}\right)=\frac{1}{3} \int_{\partial D} r^{2} \frac{\partial \Phi_{0}}{\partial n}(\mathbf{r}) d s(\mathbf{r})-\frac{2}{3}(4 \pi) \mathbf{d} \cdot \mathbf{M}_{0}^{1} \\
& +d^{2}(4 \pi) \frac{1}{3} M_{0}^{0}=\frac{1}{3} \int_{\partial D} r^{2} \frac{\partial \Phi_{0}}{\partial n}(\mathbf{r}) d s(\mathbf{r})+d^{2}(4 \pi) \frac{1}{3} M_{0}^{0} .
\end{aligned}
$$

In addition, in accordance with (99) (with $n=2$ ) and keeping $\hat{\mathbf{k}}$-terms of zeroth order, we find that

$$
\begin{aligned}
& \int_{\partial D} \frac{\partial \Phi_{2}^{(0)}}{\partial n^{\prime}}\left(\mathbf{r}^{\prime}, O^{\prime}\right) d s\left(\mathbf{r}^{\prime}\right)=\int_{\partial D} \frac{\partial \Phi_{2}^{(0)}}{\partial n}(\mathbf{r}) d s(\mathbf{r})-\frac{2}{3} \hat{\mathbf{d}} \cdot \int_{\partial D} \frac{\partial \mathbf{A}}{\partial n}(\mathbf{r}) d s(\mathbf{r}) \\
& +d^{2}(4 \pi) \frac{1}{3} M_{0}^{0}=\int_{\partial D} \frac{\partial \Phi_{2}^{(0)}}{\partial n}(\mathbf{r}) d s(\mathbf{r})+d^{2}(4 \pi) \frac{1}{3} M_{0}^{0}
\end{aligned}
$$

since as we know (from (48)), $\int_{\partial D} \frac{\partial \mathbf{A}}{\partial n}(\mathbf{r}) d s(\mathbf{r})=(4 \pi) \mathbf{M}_{0}^{1}$, which is zero with respect to the physical center. Comparing (101102), we verify the statement of the lemma. It is interesting to note that evoking (66) (on $\partial D$ ) and integrating over the scatterer, we obtain

$$
\begin{aligned}
& \frac{1}{3} \int_{\partial D} r^{2} \frac{\partial \Phi_{0}}{\partial n}(\mathbf{r}) d s(\mathbf{r})-\int_{\partial D} \frac{\partial \Phi_{2}^{(0)}}{\partial n}(\mathbf{r}) d s(\mathbf{r})=-2(4 \pi)\left(M_{0}^{0}\right)^{3} \\
& +\frac{1}{4 \pi} \int_{\partial D} \int_{\partial D}\left|\mathbf{r}-\mathbf{r}^{\prime}\right| \frac{\partial \Phi_{0}}{\partial n}(\mathbf{r}) \frac{\partial \Phi_{0}}{\partial n^{\prime}}\left(\mathbf{r}^{\prime}\right) d s(\mathbf{r}) d s\left(\mathbf{r}^{\prime}\right) .
\end{aligned}
$$

The r.h.s. of the last equation is invariant with respect to coordinate system translations and this assures alternatively that the stated equipartition of moments increases although no information about the amount of change is provided.

The next lemma is indicative for the dependence of the moments as the order of them increases.

LEMma 18. The mean values of the functions $\mathcal{H}_{2}(\hat{\mathbf{r}}, \hat{\mathbf{k}}), \mathcal{H}_{4}(\hat{\mathbf{r}}, \hat{\mathbf{k}})$ and $\mathcal{H}_{5}(\hat{\mathbf{r}}, \hat{\mathbf{k}})$ over $S^{2} \times S^{2}$ (i.e. the constant moments produced by these components) are dependent.

Proof. We define the quantities $N_{n, l}^{(m)}=\frac{1}{4 \pi} \int_{\partial D} r^{l} \frac{\partial \Phi_{n}^{(m)}}{\partial n}(\mathbf{r}) d s(\mathbf{r})$ and $N_{n, l}=$ $\frac{1}{4 \pi} \int_{\partial D} r^{l} \frac{\partial \Phi_{0}}{\partial n}(\mathbf{r}) d s(\mathbf{r})$. Let us exploit the pair (83, 26) (keeping the constant terms), and obtain the measurable quantity $\mathcal{B}_{4,0}=2 N_{2,2}^{(0)}+\frac{1}{5} N_{0,4}+N_{4,0}^{(0)}$. We need additionally the (deducible from $\mathcal{H}_{5}$ ) constant moment $\mathcal{B}_{5,0}=N_{1,4}^{(0)}+\frac{10}{3} N_{3,2}^{(0)}+N_{5,0}^{(0)}$. Using (56), we find that

$$
\mathcal{B}_{5,0}=-M_{0}^{0} N_{0,4}+\frac{10}{3} N_{3,2}^{(0)}+N_{5,0}^{(0)} .
$$

The two outcomes of Proposition 12 provide the following two results (assuming w.l.o.g. that $\mathbf{M}_{0}^{1}=0$ reflecting the specific choice of the coordinate origin):

$$
\begin{array}{r}
N_{3,2}^{(0)}+3 M_{0}^{0} N_{2,2}^{(0)}=Q N_{0,2}, \\
N_{5,0}^{(0)}+5 M_{0}^{0} N_{4,0}^{(0)}=10 Q N_{2,0}^{(0)},
\end{array}
$$


where $Q=3\left(2\left(M_{0}^{0}\right)^{3}-\mathcal{B}\right)$. We recall that $\mathcal{B}$ is the mean value of $\mathcal{H}_{2}$. Using (105, 106), we transform (104) into the form

$$
-M_{0}^{0} N_{0,4}+\frac{10}{3}\left[Q N_{0,2}-3 M_{0}^{0} N_{2,2}^{(0)}\right]+10 Q N_{2,0}^{(0)}-5 M_{0}^{0} N_{4,0}^{(0)}=\mathcal{B}_{5,0} .
$$

Inserting $\mathcal{B}_{4,0}$ into the process, we find that

$$
\begin{array}{r}
10 Q\left[\frac{1}{3} N_{0,2}+N_{2,0}^{(0)}\right]-5 M_{0}^{0} \mathcal{B}_{4,0}=\mathcal{B}_{5,0} \text { or } \\
10 Q \mathcal{B}-5 M_{0}^{0} \mathcal{B}_{4,0}=\mathcal{B}_{5,0} .
\end{array}
$$

The last equation expresses the stated dependence of the moments.

This is the point to introduce a very useful concept to the service of the reconstruction of the moments. In contrast to moments not directly accessible in measurements, there exist integral quantities involving as integrands the same normal derivatives of the components $\Phi_{n}$, which can be recovered. More precisely we introduce here the notion of double moments. These quantities are useful in the inversion scheme since they provide good estimations of surface fields in the case of star-shaped scatterers.

LEMma 19. The integrals of the normal derivatives $\frac{\partial g}{\partial n}\left(g=\Phi_{0}, \mathbf{A}, \Phi_{2}^{(0)}\right)$ over the surface $\partial D$, with respect to the measure $(\mathbf{r} \cdot \hat{\mathbf{n}}) \frac{\partial \Phi_{0}}{\partial n}$ are defined as double moments and can be expressed in terms of measured simple moments.

Proof. We start to evaluate $I_{1}=\int_{\partial D}(\mathbf{r} \cdot \hat{\mathbf{n}})\left(\frac{\partial \Phi_{0}}{\partial n}(\mathbf{r})\right)^{2} d s(\mathbf{r})$ or equivalently $I_{1}=\int_{\partial D} \mathbf{r}$. $\nabla \Phi_{0} \frac{\partial \Phi_{0}}{\partial n} d s$. After applying the operator $\mathbf{r} \cdot \nabla$ on representation (55) and restricting the result on $\partial D$ (using the well-known jump properties of surface layers), we find that

$$
\frac{1}{2} \mathbf{r} \cdot \nabla \Phi_{0}(\mathbf{r})=-\frac{1}{4 \pi} \int_{\partial D} \mathbf{r} \cdot \nabla\left(\frac{1}{\left|\mathbf{r}-\mathbf{r}^{\prime}\right|}\right) \frac{\partial \Phi_{0}}{\partial n^{\prime}}\left(\mathbf{r}^{\prime}\right) d s\left(\mathbf{r}^{\prime}\right), \quad \mathbf{r} \in \partial D
$$

or

$$
\begin{aligned}
& \frac{1}{2} \mathbf{r} \cdot \nabla \Phi_{0}(\mathbf{r})=\frac{1}{4 \pi} \int_{\partial D} \frac{1}{\left|\mathbf{r}-\mathbf{r}^{\prime}\right|} \frac{\partial \Phi_{0}}{\partial n^{\prime}}\left(\mathbf{r}^{\prime}\right) d s\left(\mathbf{r}^{\prime}\right) \\
& +\frac{1}{4 \pi} \int_{\partial D} \nabla^{\prime}\left(\frac{1}{\left|\mathbf{r}-\mathbf{r}^{\prime}\right|}\right) \cdot \mathbf{r}^{\prime} \frac{\partial \Phi_{0}}{\partial n^{\prime}}\left(\mathbf{r}^{\prime}\right) d s\left(\mathbf{r}^{\prime}\right), \quad \mathbf{r} \in \partial D .
\end{aligned}
$$

We multiply (109) with $\frac{\partial \Phi_{0}}{\partial n}$, integrate over $\partial D$, use (108) (with interchanged "primed" and "unprimed" coordinates) and (55) (on $\partial D$ ) to obtain

$$
\begin{aligned}
& \frac{1}{2} \int_{\partial D} \mathbf{r} \cdot \nabla \Phi_{0}(\mathbf{r}) \frac{\partial \Phi_{0}}{\partial n}(\mathbf{r})=\frac{1}{4 \pi} \int_{\partial D} \int_{\partial D} \frac{1}{\left|\mathbf{r}-\mathbf{r}^{\prime}\right|} \frac{\partial \Phi_{0}}{\partial n^{\prime}}\left(\mathbf{r}^{\prime}\right) \frac{\partial \Phi_{0}}{\partial n}(\mathbf{r}) d s\left(\mathbf{r}^{\prime}\right) d s(\mathbf{r}) \\
& -\frac{1}{2} \int_{\partial D} \mathbf{r}^{\prime} \cdot \nabla^{\prime} \Phi_{0}\left(\mathbf{r}^{\prime}\right) \frac{\partial \Phi_{0}}{\partial n^{\prime}}\left(\mathbf{r}^{\prime}\right) \Rightarrow \\
& I_{1}=\int_{\partial D}(\mathbf{r} \cdot \hat{\mathbf{n}})\left(\frac{\partial \Phi_{0}}{\partial n}(\mathbf{r})\right)^{2}=4 \pi M_{0}^{0} .
\end{aligned}
$$

Similarly we prove that

$$
\int_{\partial D}\left(\frac{\partial \mathbf{A}}{\partial n}(\mathbf{r})\right)(\mathbf{r} \cdot \hat{\mathbf{n}}) \frac{\partial \Phi_{0}}{\partial n}(\mathbf{r})=8 \pi \mathbf{M}_{0}^{1} .
$$


We pay attention to the double moment involving the component $\frac{\partial \Phi_{2}^{(0)}}{\partial n}(\mathbf{r}) d s(\mathbf{r})$. So we follow the above methodology to determine the integral

$$
I_{2}=\int_{\partial D}(\mathbf{r} \cdot \hat{\mathbf{n}}) \frac{\partial \Phi_{0}}{\partial n}(\mathbf{r}) \frac{\partial \Phi_{2}^{(0)}}{\partial n}(\mathbf{r}) d s(\mathbf{r}) .
$$

The starting point is now (66), which, after being subject to the application of the operator $\mathbf{r} \cdot \nabla$, provides that

$$
\begin{aligned}
& \frac{1}{2} \mathbf{r} \cdot \nabla \Phi_{2}^{(0)}(\mathbf{r})=2 \frac{r^{2}}{3}-\frac{1}{4 \pi} \int_{\partial D} \mathbf{r} \cdot \nabla\left(\frac{1}{\left|\mathbf{r}-\mathbf{r}^{\prime}\right|}\right) \frac{\partial \Phi_{2}^{(0)}}{\partial n^{\prime}}\left(\mathbf{r}^{\prime}\right) d s\left(\mathbf{r}^{\prime}\right) \\
& -\frac{1}{4 \pi} \int_{\partial D} \mathbf{r} \cdot \frac{\mathbf{r}-\mathbf{r}^{\prime}}{\left|\mathbf{r}-\mathbf{r}^{\prime}\right|} \frac{\partial \Phi_{0}}{\partial n^{\prime}}\left(\mathbf{r}^{\prime}\right) d s\left(\mathbf{r}^{\prime}\right), \quad \mathbf{r} \in \partial D .
\end{aligned}
$$

Similar manipulations as before lead to

$$
\begin{aligned}
& \frac{1}{2} \mathbf{r} \cdot \nabla \Phi_{2}^{(0)}(\mathbf{r})=-\frac{r^{2}}{3}+\frac{1}{4 \pi} \int_{\partial D} \frac{1}{\left|\mathbf{r}-\mathbf{r}^{\prime}\right|} \frac{\partial \Phi_{2}^{(0)}}{\partial n^{\prime}}\left(\mathbf{r}^{\prime}\right) d s\left(\mathbf{r}^{\prime}\right) \\
& +\frac{1}{4 \pi} \int_{\partial D} \mathbf{r}^{\prime} \cdot \nabla^{\prime}\left(\frac{1}{\left|\mathbf{r}-\mathbf{r}^{\prime}\right|}\right) \frac{\partial \Phi_{2}^{(0)}}{\partial n^{\prime}}\left(\mathbf{r}^{\prime}\right) d s\left(\mathbf{r}^{\prime}\right) \\
& +\frac{1}{4 \pi} \int_{\partial D} \mathbf{r} \cdot \frac{\mathbf{r}^{\prime}}{\left|\mathbf{r}-\mathbf{r}^{\prime}\right|} \frac{\partial \Phi_{0}}{\partial n^{\prime}}\left(\mathbf{r}^{\prime}\right) d s\left(\mathbf{r}^{\prime}\right), \quad \mathbf{r} \in \partial D
\end{aligned}
$$

and then

$$
\begin{aligned}
\int_{\partial D}(\mathbf{r} \cdot \hat{\mathbf{n}}) \frac{\partial \Phi_{2}^{(0)}}{\partial n}(\mathbf{r}) \frac{\partial \Phi_{0}}{\partial n}(\mathbf{r}) d s(\mathbf{r})=-\frac{1}{3} \int_{\partial D} r^{2} \frac{\partial \Phi_{0}}{\partial n}(\mathbf{r}) d s(\mathbf{r}) \\
\quad+\int_{\partial D} \frac{\partial \Phi_{2}^{(0)}}{\partial n}(\mathbf{r}) d s(\mathbf{r})+\frac{1}{4 \pi} \int_{\partial D} \int_{\partial D} \frac{\mathbf{r} \cdot \mathbf{r}^{\prime}}{\left|\mathbf{r}-\mathbf{r}^{\prime}\right|} \frac{\partial \Phi_{0}}{\partial n}(\mathbf{r}) \frac{\partial \Phi_{0}}{\partial n^{\prime}}\left(\mathbf{r}^{\prime}\right) d s(\mathbf{r}) d s\left(\mathbf{r}^{\prime}\right) .
\end{aligned}
$$

Next we multiply (66) (considered on $\partial D$ ), with $\frac{\partial \Phi_{0}}{\partial n}$ and integrate over the surface. We obtain

$$
\begin{aligned}
& \frac{1}{3} \int_{\partial D} r^{2} \frac{\partial \Phi_{0}}{\partial n}(\mathbf{r}) d s(\mathbf{r})-\int_{\partial D} \frac{\partial \Phi_{2}^{(0)}}{\partial n}(\mathbf{r}) d s(\mathbf{r})+2(4 \pi)\left(M_{0}^{0}\right)^{3} \\
& -\frac{1}{4 \pi} \int_{\partial D} \int_{\partial D}\left|\mathbf{r}-\mathbf{r}^{\prime}\right| \frac{\partial \Phi_{0}}{\partial n}(\mathbf{r}) \frac{\partial \Phi_{0}}{\partial n^{\prime}}\left(\mathbf{r}^{\prime}\right) d s(\mathbf{r}) d s\left(\mathbf{r}^{\prime}\right)=0 .
\end{aligned}
$$

We remark that

$$
\begin{aligned}
& \frac{1}{4 \pi} \int_{\partial D} \int_{\partial D}\left|\mathbf{r}-\mathbf{r}^{\prime}\right| \frac{\partial \Phi_{0}}{\partial n}(\mathbf{r}) \frac{\partial \Phi_{0}}{\partial n^{\prime}}\left(\mathbf{r}^{\prime}\right) d s(\mathbf{r}) d s\left(\mathbf{r}^{\prime}\right) \\
& =\frac{1}{4 \pi} \int_{\partial D} \int_{\partial D} \frac{\left|\mathbf{r}-\mathbf{r}^{\prime}\right|^{2}}{\left|\mathbf{r}-\mathbf{r}^{\prime}\right|} \frac{\partial \Phi_{0}}{\partial n}(\mathbf{r}) \frac{\partial \Phi_{0}}{\partial n^{\prime}}\left(\mathbf{r}^{\prime}\right) d s(\mathbf{r}) d s\left(\mathbf{r}^{\prime}\right)=2 \int_{\partial D} r^{2} \frac{\partial \Phi_{0}}{\partial n}(\mathbf{r}) d s(\mathbf{r}) \\
& -2 \frac{1}{4 \pi} \int_{\partial D} \int_{\partial D} \frac{\mathbf{r} \cdot \mathbf{r}^{\prime}}{\left|\mathbf{r}-\mathbf{r}^{\prime}\right|} \frac{\partial \Phi_{0}}{\partial n}(\mathbf{r}) \frac{\partial \Phi_{0}}{\partial n^{\prime}}\left(\mathbf{r}^{\prime}\right) d s(\mathbf{r}) d s\left(\mathbf{r}^{\prime}\right) .
\end{aligned}
$$

Combining (114115116), we conclude that

$$
I_{2}=\int_{\partial D}(\mathbf{r} \cdot \hat{\mathbf{n}}) \frac{\partial \Phi_{2}^{(0)}}{\partial n}(\mathbf{r}) \frac{\partial \Phi_{0}}{\partial n}(\mathbf{r}) d s(\mathbf{r})=4 \pi\left[\frac{3}{2} \mathcal{B}-\left(M_{0}^{0}\right)^{3}\right]
$$


where we recognize again the measurable "mixed"-type moment $\mathcal{B}$ with

$$
4 \pi \mathcal{B}=\frac{1}{3} \int_{\partial D} r^{2} \frac{\partial \Phi_{0}}{\partial n}(\mathbf{r}) d s(\mathbf{r})+\int_{\partial D} \frac{\partial \Phi_{2}^{(0)}}{\partial n}(\mathbf{r}) d s(\mathbf{r}) .
$$

The last lemma reveals some fundamental quantitative properties of the normal derivatives of the total field low-frequency components. First, we see immediately that the mean value of $(\mathbf{r} \cdot \hat{\mathbf{n}}) \frac{\partial \Phi_{0}}{\partial n}(\mathbf{r})$ with respect to the positive measure $\frac{\partial \Phi_{0}}{\partial n}$ is equal to 1 . In other words, $\frac{\partial \Phi_{0}}{\partial n}$ represents, in the mean, the inverse of the inner product $(\mathbf{r} \cdot \hat{\mathbf{n}})$, which provides indicative information about the curvature of the surface $\partial D$. In addition, the same weighted mean value of $\frac{1}{2}(\mathbf{r} \cdot \hat{\mathbf{n}}) \frac{\partial \mathbf{A}}{\partial n}(\mathbf{r})$ represents the physical center of the scatterer. Finally, referring to star-shape scatterers (to guarantee the positiveness of $(\mathbf{r} \cdot \hat{\mathbf{n}})$ ), combining (109110) and applying the mean value theorem, we determine the mean value of $\left(\frac{\partial \Phi_{2}^{(0)}}{\partial n} / \frac{\partial \Phi_{0}}{\partial n}\right)$ to be equal to the quantity $\left[\frac{3}{2} \mathcal{B}-\left(M_{0}^{0}\right)^{3}\right] \frac{1}{M_{0}^{0}}$. Then we may estimate the not directly accessible moment $\frac{1}{4 \pi} \int_{\partial D} \frac{\partial \Phi_{2}^{(0)}}{\partial n}(\mathbf{r}) d s(\mathbf{r})$ by the number $\left[\frac{3}{2} \mathcal{B}-\left(M_{0}^{0}\right)^{3}\right]$. This result turns out trivially to be an equality in the case of a sphere for example, but generally must be considered as an estimation produced via the application of the mean value integral calculus to the double moments regime. The following lemma opens up the possibility to give a more rigorous basis to the present formal argumentation:

Lemma 20. The normal derivative fields $g=\frac{\partial \Phi_{0}}{\partial n}, \frac{\partial \Phi_{2}^{(0)}}{\partial n}$ satisfy the integral equation

$$
2 \int_{\partial D} g(\mathbf{r}) d s(\mathbf{r})-\int_{\partial D}(\mathbf{r} \cdot \hat{\mathbf{n}})\left(\nabla_{S} \cdot \hat{\mathbf{n}}\right) g(\mathbf{r}) d s(\mathbf{r})=0
$$

where $\nabla_{S}$ is the surface gradient operator on $\partial D$.

Proof. We consider the fields

$$
\begin{aligned}
& w_{1}(\mathbf{r})=\mathbf{r} \cdot \nabla \Phi_{0}(\mathbf{r}), \quad \mathbf{r} \in R^{3} \backslash D, \\
& w_{2}(\mathbf{r})=\frac{2}{3} \mathbf{r} \cdot \nabla \Phi_{2}^{(0)}(\mathbf{r})-\frac{1}{3} \Phi_{2}^{(0)}(\mathbf{r})-\frac{1}{3} r^{2} \Phi_{0}(\mathbf{r})+\frac{2}{3}\left(M_{0}^{0}\right)^{2} \Phi_{0}(\mathbf{r}), \quad \mathbf{r} \in R^{3} \backslash D .
\end{aligned}
$$

It is straightforward to prove that these fields are harmonic outside the scatterer. In addition, in the far-field region, we apply asymptotic analysis to obtain

$$
\begin{gathered}
w_{1}(\mathbf{r})=M_{0}^{0} \frac{1}{r}+o\left(\frac{1}{r^{2}}\right), \quad r \rightarrow \infty, \\
w_{2}(\mathbf{r})=\frac{1}{3} \frac{\tilde{Q}}{r}+o\left(\frac{1}{r^{2}}\right), \quad r \rightarrow \infty,
\end{gathered}
$$

where $\tilde{Q}$ is the quantity $\left[3 \mathcal{B}-2\left(M_{0}^{0}\right)^{3}\right]$. Let us now use the Green's theorem $\int_{\partial \Omega_{R}} \frac{\partial w_{j}}{\partial n} d s$ $=\int_{\Omega_{R}} \Delta w_{j} d \mathbf{r}=0(j=1,2)$, where $\Omega_{R}$ is the region between a large sphere $B_{R}$ of radius $R$ and the scatterer $D$. The boundary $\partial \Omega_{R}$ consists of the surfaces $\partial D$ and $\partial B_{R}$. Obviously, we obtain

$$
\int_{\partial D} \frac{\partial w_{1}}{\partial n}(\mathbf{r}) d s(\mathbf{r})=-4 \pi M_{0}^{0}
$$




$$
\int_{\partial D} \frac{\partial w_{2}}{\partial n}(\mathbf{r}) d s(\mathbf{r})=-\tilde{Q}
$$

On the basis of (118119), the relations above provide that

$$
\int_{\partial D} \frac{\partial}{\partial n}\left(\mathbf{r} \cdot \nabla \Phi_{0}\right) d s=-\int_{\partial D} \frac{\partial \Phi_{0}}{\partial n} d s, \int_{\partial D} \frac{\partial}{\partial n}\left(\mathbf{r} \cdot \nabla \Phi_{2}^{(0)}\right) d s=-\int_{\partial D} \frac{\partial \Phi_{2}^{(0)}}{\partial n} d s
$$

which are written equivalently as

$$
\begin{gathered}
2 \int_{\partial D} \frac{\partial \Phi_{0}}{\partial n} d s+\int_{\partial D}(\mathbf{r} \cdot \hat{\mathbf{n}}) \frac{\partial^{2} \Phi_{0}}{\partial n^{2}} d s=0 \\
2 \int_{\partial D} \frac{\partial \Phi_{2}^{(0)}}{\partial n} d s+\int_{\partial D}(\mathbf{r} \cdot \hat{\mathbf{n}}) \frac{\partial^{2} \Phi_{2}^{(0)}}{\partial n^{2}} d s=0 .
\end{gathered}
$$

Using the decomposition of the Laplacian operator in terms of surface and normal terms $\Delta=\Delta_{S}+\left(\nabla_{S} \cdot \hat{\mathbf{n}}\right) \frac{\partial}{\partial n}+\frac{\partial^{2}}{\partial n^{2}}$ [17, we prove easily that the functions $\frac{\partial \Phi_{0}}{\partial n}$ and $\frac{\partial \Phi_{2}^{(0)}}{\partial n}$ satisfy the stated integral equation on $\partial D$. For the sake of completeness, we just mention that applying manifold differential calculus we can prove that the stated integral equation is equivalent with the relation $\int_{\partial D} \mathbf{r} \cdot \nabla_{S} g(\mathbf{r}) d s(\mathbf{r})=0$. The last equation (as the original one) is useless for a spherical scatterer, but informative for every other case.

REMARK 21. We now use the results of the preceding lemma in conjunction with the outcomes of Lemma 19 as follows:

$$
\begin{aligned}
\int_{\partial D} \frac{\partial \Phi_{2}^{(0)}}{\partial n} d s & =\frac{1}{2} \int_{\partial D}(\mathbf{r} \cdot \hat{\mathbf{n}})\left(\nabla_{S} \cdot \hat{\mathbf{r}}\right) \frac{\partial \Phi_{2}^{(0)}}{\partial n} d s \\
& =\frac{1}{2}\left[\frac{\partial \Phi_{2}^{(0)}}{\partial n} \frac{1}{\frac{\partial \Phi_{0}}{\partial n}}\right]_{\text {m.v. }} \int_{\partial D}(\mathbf{r} \cdot \hat{\mathbf{n}})\left(\nabla_{S} \cdot \hat{\mathbf{r}}\right) \frac{\partial \Phi_{0}}{\partial n} d s \\
{\left[\frac{3}{2} \mathcal{B}-\left(M_{0}^{0}\right)^{3}\right] } & \frac{1}{M_{0}^{0}} \int_{\partial D} \frac{\partial \Phi_{0}}{\partial n} d s=4 \pi\left[\frac{3}{2} \mathcal{B}-\left(M_{0}^{0}\right)^{3}\right] .
\end{aligned}
$$

Consequently we estimate again the "hidden" in measurements moment $\frac{1}{4 \pi} \int_{\partial D} \frac{\partial \Phi_{2}^{(0)}}{\partial n} d s$ with purely analytic tools, without any aid from the measurements arsenal.

The analytic determination of moments of higher order is cumbersome. Indeed the next step is the decomposition of the moment $\mathcal{B}_{4,0}=2 N_{2,2}^{(0)}+\frac{1}{5} N_{0,4}+N_{4,0}^{(0)}$ in their components. This is much more demanding than before as it involves the measure $\frac{\partial \Phi_{4}^{(0)}}{\partial n}$. The next proposition provides the double moment pertaining to this measure.

Proposition 22. The double moment $\int_{\partial D} \frac{\partial \Phi_{4}^{(0)}}{\partial n}(\mathbf{r} \cdot \hat{\mathbf{n}}) \frac{\partial \Phi_{0}}{\partial n} d s$ is analytically estimated.

Proof. The main steps of the argumentation can only be presented here since the whole proof process is very complex and elongated. The starting point is (8), corresponding to $n=4$ and actually its zeroth-order term with respect to the wave vector $\hat{\mathbf{k}}$. We "project" as usual this integral representation on $\frac{\partial \Phi_{0}}{\partial n}$, while in parallel we execute the same projection after applying to (8) the surface operator $(\mathbf{r} \cdot \nabla)$. Applying suitable jump relations and integral manipulations, we obtain the following equations:

$$
\frac{1}{5} \int_{\partial D} r^{4} \frac{\partial \Phi_{0}}{\partial n} d s-\int_{\partial D} \frac{\partial \Phi_{4}^{(0)}}{\partial n} d s-4 M_{0}^{0} \int_{\partial D} \frac{\partial \Phi_{3}^{(0)}}{\partial n} d s
$$




$$
\begin{aligned}
& -6 \frac{1}{4 \pi} \int_{\partial D} \int_{\partial D}\left|\mathbf{r}-\mathbf{r}^{\prime}\right| \frac{\partial \Phi_{0}}{\partial n} \frac{\partial \Phi_{2}^{(0)}}{\partial n^{\prime}} d s d s^{\prime} \\
& +4 M_{0}^{0} \frac{1}{4 \pi} \int_{\partial D} \int_{\partial D}\left|\mathbf{r}-\mathbf{r}^{\prime}\right|^{2} \frac{\partial \Phi_{0}}{\partial n} \frac{\partial \Phi_{0}}{\partial n^{\prime}} d s d s^{\prime} \\
& -\frac{1}{4 \pi} \int_{\partial D} \int_{\partial D}\left|\mathbf{r}-\mathbf{r}^{\prime}\right|^{3} \frac{\partial \Phi_{0}}{\partial n} \frac{\partial \Phi_{0}}{\partial n^{\prime}} d s d s^{\prime}=0, \\
& \int_{\partial D}(\mathbf{r} \cdot \hat{\mathbf{n}}) \frac{\partial \Phi_{4}^{(0)}}{\partial n} \frac{\partial \Phi_{0}}{\partial n} d s=\frac{4}{5} \int_{\partial D} r^{4} \frac{\partial \Phi_{0}}{\partial n} d s \\
& +\int_{\partial D} \frac{\partial \Phi_{4}^{(0)}}{\partial n} d s-6 \frac{1}{4 \pi} \int_{\partial D} \int_{\partial D}\left|\mathbf{r}-\mathbf{r}^{\prime}\right| \frac{\partial \Phi_{0}}{\partial n} \frac{\partial \Phi_{2}^{(0)}}{\partial n^{\prime}} d s d s^{\prime} \\
& -6 \frac{1}{4 \pi} \int_{\partial D} \int_{\partial D} \mathbf{r}^{\prime} \cdot \frac{\left(\mathbf{r}-\mathbf{r}^{\prime}\right)}{\left|\mathbf{r}-\mathbf{r}^{\prime}\right|} \frac{\partial \Phi_{0}}{\partial n} \frac{\partial \Phi_{2}^{(0)}}{\partial n^{\prime}} d s d s^{\prime} \\
& -8 \frac{1}{4 \pi} \int_{\partial D} r^{2} \frac{\partial \Phi_{0}}{\partial n} d s \int_{\partial D} \frac{\partial \Phi_{2}^{(0)}}{\partial n^{\prime}} d s^{\prime} \\
& -\frac{3}{2} \frac{1}{4 \pi} \int_{\partial D} \int_{\partial D}\left|\mathbf{r}-\mathbf{r}^{\prime}\right|^{3} \frac{\partial \Phi_{0}}{\partial n} \frac{\partial \Phi_{0}}{\partial n^{\prime}} d s d s^{\prime} .
\end{aligned}
$$

In addition, we project (66) onto $\frac{\partial \Phi_{2}^{(0)}}{\partial n}$ to obtain

$$
\begin{array}{r}
\frac{1}{3} \int_{\partial D} r^{2} \frac{\partial \Phi_{2}^{(0)}}{\partial n} d s-\int_{\partial D} \int_{\partial D}\left|\mathbf{r}-\mathbf{r}^{\prime}\right|^{-1} \frac{\partial \Phi_{2}^{(0)}}{\partial n} \frac{\partial \Phi_{2}^{(0)}}{\partial n^{\prime}} d s d s^{\prime} \\
+2\left(M_{0}^{0}\right)^{2} \int_{\partial D} \frac{\partial \Phi_{2}^{(0)}}{\partial n} d s-\frac{1}{4 \pi} \int_{\partial D} \int_{\partial D}\left|\mathbf{r}-\mathbf{r}^{\prime}\right| \frac{\partial \Phi_{2}^{(0)}}{\partial n} \frac{\partial \Phi_{2}^{(0)}}{\partial n^{\prime}} d s d s^{\prime}=0 .
\end{array}
$$

We also project (112) onto $\frac{\partial \Phi_{2}^{(0)}}{\partial n}$ to give an alternative representation to the "tough" integral $\int_{\partial D} \mathbf{r}^{\prime} \cdot \frac{\left(\mathbf{r}-\mathbf{r}^{\prime}\right)}{\left|\mathbf{r}-\mathbf{r}^{\prime}\right|} \frac{\partial \Phi_{0}}{\partial n} \frac{\partial \Phi_{2}^{(0)}}{\partial n^{\prime}} d s d s^{\prime}$, which already appeared in (125), in order to eliminate it. Finally, eliminating $\int_{\partial D} \int_{\partial D}\left|\mathbf{r}-\mathbf{r}^{\prime}\right|^{3} \frac{\partial \Phi_{0}}{\partial n} \frac{\partial \Phi_{0}}{\partial n^{\prime}} d s d s^{\prime}$ from (124, 125) and using (126) together with the first interrelation outcome of Proposition 12 (to absorb $\Phi_{3}$ ), we obtain

$$
\begin{aligned}
& \frac{1}{4 \pi} \int_{\partial D}(\mathbf{r} \cdot \hat{\mathbf{n}}) \frac{\partial \Phi_{4}^{(0)}}{\partial n} \frac{\partial \Phi_{0}}{\partial n} d s=\frac{5}{2} \mathcal{B}_{4,0}-3 \frac{1}{4 \pi} \int_{\partial D}(\mathbf{r} \cdot \hat{\mathbf{n}})\left(\frac{\partial \Phi_{2}^{(0)}}{\partial n}\right)^{2} d s \\
& -12\left(M_{0}^{0}\right)^{2}\left[3 \mathcal{B}-2 \frac{1}{4 \pi} \int_{\partial D} \frac{\partial \Phi_{2}^{(0)}}{\partial n} d s\right]+6 Q\left(M_{0}^{0}\right)^{2} \\
& +24 M_{0}^{0}\left|\mathbf{M}_{0}^{1}\right|^{2}-8 \frac{1}{4 \pi}\left(\int_{\partial D} \frac{\partial \Phi_{2}^{(0)}}{\partial n} d s\right)\left[3 \mathcal{B}-3 \frac{1}{4 \pi} \int_{\partial D} \frac{\partial \Phi_{2}^{(0)}}{\partial n} d s\right] .
\end{aligned}
$$

We verify that (127) expresses the aimed double moment in terms of moments of lower order that have already been measured or analytically estimated. Actually, among the terms participating in the r.h.s. of (127), only the second one merits some special care and this can be estimated as $-\frac{3}{4 \pi}\left[\frac{\partial \Phi_{2}^{(0)}}{\partial n} \frac{1}{\frac{\partial \Phi_{0}}{\partial n}}\right]_{\text {m.v. }}^{2} \int_{\partial D}(\mathbf{r} \cdot \hat{\mathbf{n}})\left(\frac{\partial \Phi_{0}}{\partial n}\right)^{2} d s=-\frac{3}{4} \frac{\tilde{Q}^{2}}{M_{0}^{0}}$. Working 
as before, we estimate the moment $N_{4,0}^{(0)}=\frac{1}{4 \pi} \int_{\partial D} \frac{\partial \Phi_{4}^{(0)}}{\partial n} d s$ to be just the r.h.s. $\mathcal{L}$ of (127).

REMARK 23. The measured moment $\mathcal{B}_{4,0}=2 N_{2,2}^{(0)}+\frac{1}{5} N_{0,4}+N_{4,0}^{(0)}$ is decomposed as follows: The term $N_{4,0}^{(0)}$ has just been defined in Proposition 22 while $N_{2,2}^{(0)}$ is estimated as

$$
\begin{aligned}
& \frac{1}{4 \pi} \int_{\partial D} r^{2} \frac{\partial \Phi_{2}^{(0)}}{\partial n} d s=\left[\frac{\partial \Phi_{2}^{(0)}}{\partial n} \frac{1}{\frac{\partial \Phi_{0}}{\partial n}}\right]_{\mathrm{m} . \mathrm{v} \cdot} \frac{1}{4 \pi} \int_{\partial D} r^{2} \frac{\partial \Phi_{0}}{\partial n} d s \\
& =\left[\frac{3}{2} \mathcal{B}-\left(M_{0}^{0}\right)^{3}\right] \frac{1}{M_{0}^{0}}\left[-\frac{3}{2} \mathcal{B}+3\left(M_{0}^{0}\right)^{3}\right] .
\end{aligned}
$$

Then the remaining moment $N_{0,4}=\frac{1}{4 \pi} \int_{\partial D} r^{4} \frac{\partial \Phi_{0}}{\partial n} d s$, pertaining to the Rayleigh component $\Phi_{0}$, can be appropriately determined.

A similar deconstruction can be executed in more intrinsic moments. It is not possible to present extensively all the cases but we present the following indicative steps, omitting the lengthy proofs, which in principle could be reminiscent of techniques already well exhibited in this work.

LEMma 24. (i) The vector moment $\mathbf{B}_{1}=\frac{1}{4 \pi}\left\{\frac{3}{5} \int_{\partial D} \frac{\partial \mathbf{A}_{3}}{\partial n} d s+\int_{\partial D} r^{2} \frac{\partial \mathbf{A}}{\partial n}\right\} d s$ is metrizable and equal to

$$
\mathbf{B}_{1}=\frac{1}{3} \sum_{i} \sum_{j} \hat{\mathcal{H}}_{3}^{s y m}\left(\hat{\mathbf{r}}_{i} ; \hat{\mathbf{k}}_{j}\right) \hat{\mathbf{k}}_{j}-\frac{2}{5} \sum_{m=-3}^{3} \frac{(3-|m|) !}{(3+|m|) !} \sum_{j}\left[\overline{Y_{3}^{m}\left(\hat{\mathbf{k}}_{j}\right)} \hat{\mathbf{k}}_{j}\right] \mathcal{M}_{0}^{(3, m)}
$$

where $\hat{\mathbf{r}_{i}}, \hat{\mathbf{k}}_{j}$ stand for the orthonormal cartesian basis in $R^{3}$.

(ii) The vector moment $\mathbf{B}_{2}=\frac{1}{4 \pi}\left\{3 \int_{\partial D} \mathbf{r} \frac{\partial \Phi_{2}^{(0)}}{\partial n} d s+\frac{3}{5} \int_{\partial D} r^{2} \mathbf{r} \frac{\partial \Phi_{0}}{\partial n}\right\} d s$ is measured to be:

$$
\begin{aligned}
\mathbf{B}_{2}=- & \sum_{i} \tilde{\mathcal{H}}_{3}^{a n t}\left(\hat{\mathbf{r}_{i}} ; \hat{\mathbf{k}}\right) \hat{\mathbf{r}_{i}}-2 \sum_{m=-2}^{2} \frac{(2-|m|) !}{(2+|m|) !} \overline{Y_{2}^{m}(\hat{\mathbf{k}})} \int_{\partial D} r^{2} Y_{2}^{m}(\hat{\mathbf{r}}) \frac{\partial \mathbf{A}}{\partial n} d s(\mathbf{r}) \\
& -\frac{2}{5} \sum_{m=-3}^{3} \frac{(3-|m|) !}{(3+|m|) !} \sum_{i}\left[\overline{Y_{3}^{m}\left(\hat{\mathbf{r}}_{i}\right)} \hat{\mathbf{r}}_{i}\right] \mathcal{M}_{0}^{(3, m)},
\end{aligned}
$$

for an arbitrary excitation direction $\hat{\mathbf{k}}$. The involved moments $\int_{\partial D} r^{2} Y_{2}^{m}(\hat{\mathbf{r}}) \frac{\partial \mathbf{A}}{\partial n} d s(\mathbf{r})$ are constructed in Remark 13 .

Proposition 25. The double moment $\frac{1}{4 \pi} \int_{\partial D}(\mathbf{r} \cdot \hat{\mathbf{n}}) \frac{\partial \mathbf{A}_{3}}{\partial n} \frac{\partial \Phi_{0}}{\partial n} d s$ is equal to $\frac{5}{3} \mathbf{B}_{1}+5 \mathbf{B}_{2}-$ $15 \mathcal{B} \frac{1}{M_{0}^{0}} \mathbf{M}_{0}^{1}$.

Following the usual method, we infer that the moment $\frac{1}{4 \pi} \int_{\partial D} \frac{\partial \mathbf{A}_{3}}{\partial n} d s$ is estimated by the quantity $\frac{5}{3} \mathbf{B}_{1}+5 \mathbf{B}_{2}-15 \mathcal{B} \frac{1}{M_{0}^{0}} \mathbf{M}_{0}^{1}$. Then it is possible to obtain an appropriate evaluation of the moment $\frac{1}{4 \pi} \int_{\partial D} r^{2} \frac{\partial \mathbf{A}}{\partial n} d s$ as the quantity $9 \mathcal{B} \frac{1}{M_{0}^{0}} \mathbf{M}_{0}^{1}-3 \mathbf{B}_{2}$. 
7. Discussion. In the present work, we investigated all possible ways to determine the surface moments of the scattering process, which are included in the far-field pattern. We paid attention first to establishing a systematic method for the determination of the low-frequency components of the scattering amplitude in a stable manner, exploiting the data for several wave numbers in the low-frequency regime. These components contain the moments, which constitute integrals over the scatterer's surface with integrands built over the components of the low-frequency expansion of the total acoustic field. The principal concern of this work has been the settlement of a method aiming at extracting the moments from the far field. The moments pertaining to the fundamental total field component (the Rayleigh component) had already been presented in a series of introductory papers, which brought into light a novel method for the solution of the inverse scattering problem. The moments referring to the Rayleigh component are not influenced by the excitation direction. In contrast, the moments corresponding to higher-order components of the total field depend strongly on the direction of the incident plane wave. In any case, in the present work we prove that the moments are divided into two categories with different characteristics. The first class involves the moments that are deducible from the measurements (and the present work investigates this deduction) while the second class contains all the other moments that build measurable superstructures, which can not be decomposed via the data information. Nevertheless, these inaccessible moments can be estimated analytically via the integral equation calculus of double moments, leading to resolving of this involvement of moments. This methodology is demanding and becomes more complicated as the order of the total field component increases. As will be apparent in the subsequent relative work, insisting on the linear version of the method renders inevitable the evocation of this analytic complex calculus. However, it is possible to detour this analysis, drop the not directly accessible in measurements moments and work only with the first class of moments, for several interesting cases. The price is to renovate the method in a nonlinear form, confronting both the case of polynomial scatterers as well as the case of scatterers estimated by polynomial manifolds. Working in the old or new framework, it is necessary to define with stability the path originating from the data and leading to the determination of the moments and this is the outcome of the present work.

\section{REFERENCES}

[1] Charalambopoulos, A., Inverse scattering for an acoustically soft scatterer in the low-frequency region, Int. J. Engrg. Sci., 33, 4, (1995), 599-609. MR1314301 (95m:76059)

[2] Charalambopoulos, A., Kiriaki, K., A method for solving the inverse elastic scattering problem via low-frequency moments, Wave Motion, 18 (1993), 213-226. MR.1256478 (95f:73032)

[3] Charalambopoulos, A., Dassios, G., Inverse scattering via low-frequency moments, J. Math. Phys., 33, (1992), 4201-4216. MR.1191780 (93j:35051)

[4] Charalambopoulos, A., The reconstruction of the surface of scatterers with continuous curvature via low-frequency moments, IMA Journal of Applied Mathematics, 54, (1995), 171-201. MR1334459 (96c:35192)

[5] Apostolopoulos, T., Dassios, G. A parallel algorithm for solving the inverse scattering moment problem, Journal of Computational and Applied Mathematics, 42, (1992), 63-77. MR 1181581

[6] Charalambopoulos, A., An analytic algorithm for shape reconstruction from low-frequency moments, accepted for publication in Journal of Mathematical Physics, 2011.

[7] Billingham, J., King, A.C., Wave Motion, Cambridge University Press, 2000. MR1811404 (2001k:35001) 
[8] Kleinman, R.E., The Rayleigh region, PROC. IEEE, 53, (1965), 848-856.

[9] Kleinman, R.E., The Dirichlet problem for the Helmholtz Equation, Arch. Rat. Mech. Analysis, 18, (1965), 205-229. MR0172602 (30:2821)

[10] Kleinman, R.E., Far-Field scattering at low-frequencies, Appl. Sci. Res., 18, (1967), 1-8.

[11] Muller, C., Radiation Patterns and Radiation Fields, J. Rat. Mech. and Anal., 4, (1955), 235-245. MR0069026(16:978b)

[12] Charalambopoulos, A., Kiriaki, K., Characterization of Functions as Radiation Patterns in Linear Elasticity, Mathematical Methods in the Applied Sciences, 15, (1992), 547-558. MR1184322 (93j:73030)

[13] Kirsch, A., Characterization of the shape of a scattering obstacle using the spectra data of the far field operator, Inverse Problems, 15, (1998), 413-419. MR.1662460 (99k:35193)

[14] Groetsch, C.W., Stable Approximate Evaluation of Unbounded Operators, Springer-Verlag, 2007. MR2268011(2008a:47022)

[15] Morse, Philip M., Feshbach, Herman, Methods of Theoretical Physics, 2 Volumes, McGraw-Hill Book Co., 1953. MR0059774 (15:583h)

[16] Colton, D., Kress, R., Integral equation methods in scattering theory, John Wiley and Sons, 1983. MR700400 (85d:35001)

[17] Nedelec, J.C., Acoustic and Electromagnetic Equations, Integral Representations for Harmonic Problems, Applied Mathematical Sciences, Vol. 44, Springer-Verlag, 2000. MR1822275 (2002c:35003) 